\title{
THE CALIFORNIA PLANET SURVEY IV: A PLANET ORBITING THE GIANT STAR HD 145934 AND UPDATES TO SEVEN SYSTEMS WITH LONG-PERIOD PLANETS*
}

\author{
Y. Katherina Feng ${ }^{1,4}$, Jason T. Wright ${ }^{1}$, Benjamin Nelson ${ }^{1}$, Sharon X. Wang ${ }^{1}$, Eric B. Ford ${ }^{1}$, \\ GeOFFrey W. MARCY ${ }^{2}$, HowARD ISAACSON ${ }^{2}$, AND ANDreW W. HOWARD ${ }^{3}$ \\ ${ }^{1}$ Center for Exoplanets and Habitable Worlds, Department of Astronomy \& Astrophysics, 525 Davey Lab, \\ The Pennsylvania State University, University Park, PA 16802, USA; astrowright@gmail.com \\ 2 Department of Astronomy, University of California, Berkeley, CA 94720-3411, USA \\ ${ }^{3}$ Institute for Astronomy, University of Hawaii, 2680 Woodlawn Drive, Honolulu, HI 96822, USA \\ Received 2014 August 11; accepted 2014 November 26; published 2015 February 5
}

\begin{abstract}
We present an update to seven stars with long-period planets or planetary candidates using new and archival radial velocities from Keck-HIRES and literature velocities from other telescopes. Our updated analysis better constrains orbital parameters for these planets, four of which are known multi-planet systems. HD $24040 b$ and HD $183263 c$ are super-Jupiters with circular orbits and periods longer than $8 \mathrm{yr}$. We present a previously unseen linear trend in the residuals of HD 66428 indicative of an additional planetary companion. We confirm that GJ 849 is a multi-planet system and find a good orbital solution for the $c$ component: it is a $1 M_{\text {Jup }}$ planet in a $15 \mathrm{yr}$ orbit (the longest known for a planet orbiting an M dwarf). We update the HD 74156 double-planet system. We also announce the detection of HD $145934 b$, a $2 M_{\text {Jup }}$ planet in a $7.5 \mathrm{yr}$ orbit around a giant star. Two of our stars, HD 187123 and HD 217107, at present host the only known examples of systems comprising a hot Jupiter and a planet with a well constrained period greater than $5 \mathrm{yr}$, and with no evidence of giant planets in between. Our enlargement and improvement of long-period planet parameters will aid future analysis of origins, diversity, and evolution of planetary systems.
\end{abstract}

Key words: planetary systems - stars: individual (HD 145934, GJ 849) - techniques: radial velocities

Supporting material: machine-readable table

\section{INTRODUCTION}

\subsection{Background}

The possibility of "Earth 2.0," and especially another planet that hosts life, drives much of the search for exoplanets. As of 2014 November, efforts over two decades have uncovered more than 1400 planets and almost 4000 planetary candidates (Han et al. 2014; Burke et al. 2014; exoplanets.org). The variety of discoveries, from lone Jupiter-mass planets in few-day orbits to packed systems with multiple planets that fit within Mercury's orbit, raises a significant question as to the nature of our solar system: are we unique?

To search for analogs of the solar system, we target multiplanet systems and long-period giant planets, reminiscent of our own outer solar system. Because we seek planets with orbits of at least a few hundred days, the radial velocity (RV) method of exoplanet detection is advantageous (e.g., Wright \& Gaudi 2013; Butler et al. 1996; Mayor \& Queloz 1995). The RV method is the longest running, with multiple surveys studying thousands of stars. In our study, we utilize up-to-date velocities from Keck observatory's High Resolution Echelle Spectrometer (HIRES; Vogt et al. 1994) and complementary, published velocities from other telescopes, where available.

So far, the number of planets discovered by RVs with periods greater than 1000 days is 103 , only 16 of which have periods longer than 3000 days. This is in contrast to the 336 such

\footnotetext{
* Based in part on observations obtained at the W. M. Keck Observatory, which is operated by the University of California and the California Institute of Technology.

4 Also at Department of Astronomy \& Astrophysics, 1156 High Street, MS: UCO/LICK, University of California, Santa Cruz, CA 95064, USA.
}

planets with shorter periods. ${ }^{5}$ Our intent is to enlarge this sample of long-period planets to search for planetary systems with Jupiter analogs. Of these 103 planets, 31 are in multiplanet systems. The study of multi-planet systems addresses planetary formation, migration, and dynamics. Having a large sample can also contribute to the understanding of the evolution and lifetime of stable planetary systems. Studies can examine the orbital eccentricities and perform dynamic simulations and probe migration.

For the purposes of this discussion, we follow Wang et al. (2012) and define a Jupiter analog as a planet with $P>8 \mathrm{yr}$, $4>M \sin (i)>0.5 M_{\mathrm{Jup}}$, and $e<0.3$, but we also adopt an upper period limit of $P<16$ yr. Of the confirmed RV planets, only 13 planets fit the above criteria (Han et al. 2014). Another motivation for studying systems with long-period Jupiter analogs is the role such a Jovian planet may play in the habitability of an Earth-like planet in the same system. Wetherill (1994) argued that Jupiter acts as a shield that deflects comets originating from the Oort Cloud or Kuiper Belt, protecting the inner solar system. Without Jupiter, Wetherill (1994) suggested an increase in the frequency of cometary impacts on Earth by 1000-10,000 times the present-day value. Multi-planet systems serve not only as examples of planet-planet interaction but also as models for planet-comet dynamics.

\subsection{Plan}

Section 2 gives an outline of the steps taken for characterizing the planetary systems. In Section 3, we describe the planetary systems orbiting seven stars. Each of these systems already has at least one planet known and exhibits RV residuals indicative

\footnotetext{
5 We follow Han et al. (2014), who adopt a upper limit on minimum mass of 24 times the mass of Jupiter.
} 
Table 1

Summary of Radial Velocity Data (Number of Observations) and Mean Uncertainties

\begin{tabular}{|c|c|c|c|c|c|c|}
\hline & Instrument & $\begin{array}{l}\text { Mean Unc. } \\
\left(\mathrm{m} \mathrm{s}^{-1}\right)\end{array}$ & $N_{\text {obs }}$ & $\begin{array}{c}\text { Span } \\
(\mathrm{yr})\end{array}$ & $N_{\text {new }}$ & $\begin{array}{l}\text { Offset from Instrument } 1 \\
\qquad\left(\mathrm{~m} \mathrm{~s}^{-1}\right)\end{array}$ \\
\hline \multicolumn{7}{|c|}{ HD $24040 *$} \\
\hline 1 & SOPHIE & 4.27 & 13 & $2008-2010$ & $\ldots$ & $\ldots$ \\
\hline 2 & ELODIE & 11.48 & 47 & $1997-2005$ & $\ldots$ & $-53.19 \pm 4.37$ \\
\hline 3 & HIRES (pre-upgrade) & 1.35 & 20 & 1998-2004 & $\ldots$ & $-44.73 \pm 3.80$ \\
\hline$\underline{4}$ & HIRES (post-upgrade) & 1.56 & 27 & 2004-late 2013 & 22 & $-34.28 \pm 1.69$ \\
\hline \multicolumn{7}{|c|}{ HD 66428. Jitter $=3 \mathrm{~m} \mathrm{~s}^{-1}$} \\
\hline 1 & HIRES (pre-upgrade) & 1.22 & 22 & 2000-2004 & $\ldots$ & $\ldots$ \\
\hline$\underline{2}$ & HIRES (post-upgrade) & 1.05 & 33 & 2004-late 2013 & 26 & $2.8 \pm 1.8$ \\
\hline \multicolumn{7}{|c|}{ HD $74156^{*}$} \\
\hline 1 & CORALIE & 8.52 & 44 & $2001-2003$ & $\cdots$ & $\cdots$ \\
\hline 2 & HRS & 8.34 & 82 & 2004-2007 & $\ldots$ & $-32.5 \pm 3.07$ \\
\hline 3 & ELODIE & 12.74 & 51 & $1998-2003$ & $\ldots$ & $32.16 \pm 2.82$ \\
\hline 4 & HIRES (pre-upgrade) & 1.99 & 9 & $2001-2004$ & $\ldots$ & $47.02 \pm 2.6$ \\
\hline 5 & HIRES (post-upgrade) & 2.87 & 43 & 2004-late 2013 & 31 & $66.73 \pm 2.6$ \\
\hline \multicolumn{7}{|c|}{ HD 145934. Jitter $=7.5 \mathrm{~m} \mathrm{~s}^{-1}$} \\
\hline 1 & HIRES (pre-upgrade) & 1.22 & 44 & 1997-2004 & $\ldots$ & $\ldots$ \\
\hline 2 & HIRES (post-upgrade) & 1.00 & 31 & 2004-early 2014 & 75 & $14.99 \pm 9.70$ \\
\hline \multicolumn{7}{|c|}{ HD 183263. Jitter $=3.2 \mathrm{~m} \mathrm{~s}^{-1}$} \\
\hline 1 & HIRES (pre-upgrade) & 1.6 & 31 & 2001-2004 & $\ldots$ & $\ldots$ \\
\hline 2 & HIRES (post-upgrade) & 1.23 & 11 & 2004-mid-2013 & 24 & $4.04 \pm 4.4$ \\
\hline \multicolumn{7}{|c|}{ HD 187123. Jitter $=2.23 \mathrm{~m} \mathrm{~s}^{-1}$} \\
\hline 1 & HIRES (pre-upgrade) & 1.22 & 64 & 1997-2004 & $\ldots$ & $\ldots$ \\
\hline 2 & HIRES (post-upgrade) & 1.19 & 46 & 2004-mid-2013 & 40 & $-1.58 \pm 0.88$ \\
\hline \multicolumn{7}{|c|}{ HD $217107 *$} \\
\hline 1 & Hamilton & 4.709 & 121 & 1998-2007 & $\ldots$ & $\ldots$ \\
\hline 2 & CORALIE & 9.175 & 63 & 1998-1999 & $\ldots$ & $-402.3 \pm 1.74$ \\
\hline 3 & HIRES (pre-upgrade) & 1.414 & 63 & 1998-2004 & $\ldots$ & $17.6 \pm 1.38$ \\
\hline$\underline{4}$ & HIRES (post-upgrade) & 1.022 & 68 & 2004-late 2013 & 31 & $25.68 \pm 1.87$ \\
\hline \multicolumn{7}{|c|}{ GJ 849. Jitter $=3 \mathrm{~m} \mathrm{~s}^{-1}$} \\
\hline 1 & HIRES (pre-upgrade) & 3.19 & 24 & 1997-2004 & $\ldots$ & $\ldots$ \\
\hline 2 & HIRES (post-upgrade) & 1.48 & 58 & 2004-late 2013 & 3 & $-4.24 \pm 2.24$ \\
\hline 3 & HARPS & 1.05 & 35 & 2003-2008 & $\ldots$ & $18.36 \pm 2.1$ \\
\hline
\end{tabular}

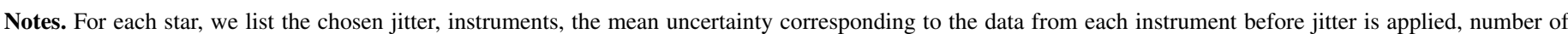

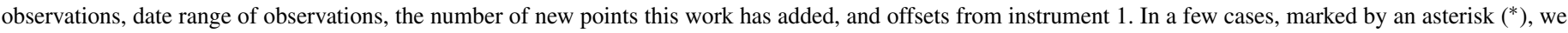
add jitter instrument-by-instrument. We split data from HIRES by the 2004 upgrade. For references of data, see the text.

of an outer companion. Additionally, each can have its planetary orbits significantly refined with our new velocities from Keck, and in some cases we show that an outer, decade-long planetary orbit has finally completed. We present a summary of the radial velocity data, mean uncertainties, and telescope offsets in Table 1. Table 2 lists the stellar parameters of the target stars. Table 3 lists the orbital parameters of the planets presented in this paper. Figures of RV curves and residuals to fits for each system are available after the text. Section 4 presents an analysis of a new planet, HD $145934 b$. We discuss our findings and future prospects in Section 5.

\section{METHODOLOGY}

\subsection{Radial Velocity Sources and Analysis}

We combine previously published data from other telescopes to complement the time span and quantity of Keck-HIRES observations obtained by the California Planet Survey (Howard et al. 2010; Johnson et al. 2010; Wright et al. 2011) for many purposes, including as part of the $\eta_{\oplus}$ survey (Howard et al.
2009, 2010, 2011a, 2011b). At the time of the first confirmed RV planet, 51 Pegasi $b$ (Mayor \& Queloz 1995), several surveys were underway and actively monitoring stars for the signs of planets (e.g., Cochran \& Hatzes 1994; Fischer et al. 2014). The discovery team for 51 Pegasi used the ELODIE spectrograph (Baranne et al. 1996), which was part of the Northern Extrasolar Planet Search until the SOPHIE spectrograph (Bouchy \& Sophie Team 2006) replaced it in 2006. The CORALIE spectrograph (e.g., Queloz et al. 2000) was situated in Chile as part of the Southern Sky extrasolar Planet search Programme. It has been joined by HARPS (Mayor et al. 2003), also located in Chile. We make use of literature data from all four spectrographs in this work. Other data come from High Resolution Spectrograph (HRS) of the Hobby-Eberly Telescope (Tull 1998), the Tull Spectrograph at the $2.7 \mathrm{~m}$ telescope of McDonald Observatory (Tull et al. 1995), and the Hamilton spectrograph at Lick Observatory (Vogt 1987).

To analyze and fit the data, we use the Wright \& Howard (2009) RVLIN package written in IDL that naturally handles multiplanet systems using data from multiple telescopes in 
Table 2

List of Stellar Parameters

\begin{tabular}{|c|c|c|c|c|c|c|c|c|c|c|c|c|}
\hline Name & $\begin{array}{l}\text { R.A. } \\
\text { (2) }\end{array}$ & $\begin{array}{l}\text { Decl. } \\
\text { (3) }\end{array}$ & $\begin{array}{c}\text { Sp. Ty. } \\
\text { (4) }\end{array}$ & (5) & $\begin{array}{c}B-V \\
(6)\end{array}$ & $\begin{array}{c}\text { Dist } \\
(\mathrm{pc}) \\
(7)\end{array}$ & $\begin{array}{l}T_{\text {eff }} \\
(\mathrm{K}) \\
(8)\end{array}$ & $\begin{array}{c}\log (g) \\
(\mathrm{cgs}) \\
(9)\end{array}$ & $\begin{array}{c}{[\mathrm{Fe} / \mathrm{H}]} \\
(\mathrm{dex}) \\
(10)\end{array}$ & $\begin{array}{c}v \sin (i) \\
\left(\mathrm{km} \mathrm{s}^{-1}\right) \\
(11)\end{array}$ & $\begin{array}{c}M_{\star} \\
\left(M_{\odot}\right) \\
(12)\end{array}$ & $\begin{array}{c}R_{\star} \\
\left(R_{\odot}\right) \\
(13)\end{array}$ \\
\hline HD 24040 & 035022.9 & +172834.9 & G0 & 7.5 & 0.65 & $46.6(1.6)$ & $5853(44)$ & $4.361(60)$ & $0.206(30)$ & $2.39(50)$ & $1.18(10)$ & $1.154(39)$ \\
\hline HD 66428 & 080328.7 & -010945.7 & G5 & 8.3 & 0.71 & $54.9(3.2)$ & $5752(44)$ & $4.490(60)$ & $0.310(30)$ & $0.00(50)$ & $1.061(63)$ & $0.980(34)$ \\
\hline HD 74156 & 084225.1 & +0434 41.1 & G0 & 7.6 & 0.58 & $64.4(2.2)$ & $6068(44)$ & $4.259(60)$ & $0.131(30)$ & $4.32(50)$ & $1.238(42)$ & $1.345(44)$ \\
\hline HD 145934 & 161309.9 & +131422.1 & K0 & 8.5 & 1.05 & $\ldots$ & $\ldots$ & $3.23(6)$ & & . & $1.748(105)$ & $5.38(44)$ \\
\hline HD 183263 & 192824.6 & +082128.9 & G2IV & 7.9 & 0.68 & $55.1(2.8)$ & $5936(44)$ & $4.403(60)$ & $0.302(30)$ & $1.56(50)$ & $1.121(52)$ & $1.117(38)$ \\
\hline HD 187123 & 194658.1 & +342510.3 & G5V & 7.8 & 0.66 & $48.3(1.2)$ & $5815(44)$ & $4.359(60)$ & $0.121(30)$ & $2.15(50)$ & $1.037(25)$ & $1.143(39)$ \\
\hline HD 217107 & 225815.5 & -022343.4 & G8 & 6.2 & 0.74 & $19.86(15)$ & $5704(44)$ & $4.541(60)$ & $0.389(30)$ & $0.00(50)$ & $1.108(43)$ & $1.500(30)$ \\
\hline GJ 849 & 220940.3 & -043826.6 & M3.5V & 10.4 & 1.5 & $9.10(17)$ & $3601(19)^{\mathrm{a}}$ & $\ldots$ & $0.31(12)^{\mathrm{a}}$ & & $0.490(49)^{\mathrm{a}}$ & \\
\hline
\end{tabular}

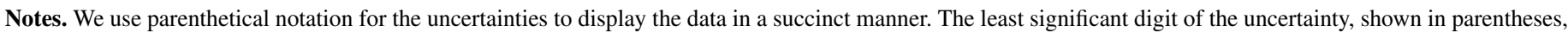

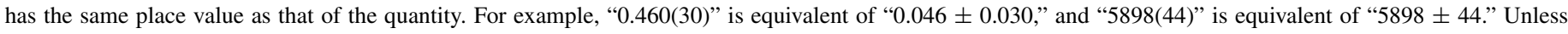

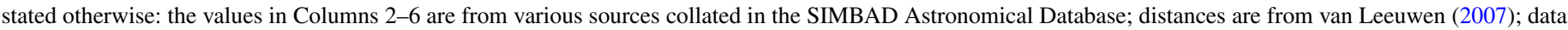

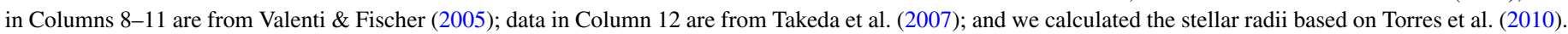
${ }^{\text {a }}$ For GJ 849, $T_{\text {eff }}$ is from Rojas-Ayala et al. (2012), [Fe/H] is from Terrien et al. (2012), while $M_{\star}$ is from Montet et al. (2014).

Table 3

List of Keplerian Orbital Parameters

\begin{tabular}{|c|c|c|c|c|c|c|c|c|}
\hline Name & & $\begin{array}{c}P \\
\text { (days) }\end{array}$ & $\begin{array}{c}T_{\mathrm{p}} \\
(\mathrm{BJD}-2440000)\end{array}$ & $e$ & $\begin{array}{l}\omega \\
\left({ }^{\circ}\right)\end{array}$ & $\begin{array}{c}K \\
\left(\mathrm{~m} \mathrm{~s}^{-1}\right)\end{array}$ & $\begin{array}{l}M \sin i \\
\left(M_{\mathrm{Jup}}\right)\end{array}$ & $\begin{array}{c}a \\
(\mathrm{AU})\end{array}$ \\
\hline HD 24040 & $b^{\mathrm{a}}$ & $3490 \pm 25$ & $16670 \pm 240$ & $0.047 \pm 0.020$ & $67 \pm 24$ & $51.8 \pm 1.6$ & $4.10 \pm 0.12$ & $4.637 \pm 0.067$ \\
\hline HD 66428 & $b^{\mathrm{b}}$ & $2293.9 \pm 6.4$ & $12278 \pm 16$ & $0.440 \pm 0.013$ & $180.4 \pm 2.6$ & $52.6 \pm 1.1$ & $3.194 \pm 0.060$ & $3.471 \pm 0.069$ \\
\hline \multirow[t]{2}{*}{ HD 74156} & $b$ & $51.6385 \pm 0.0015$ & $10793.39 \pm 0.11$ & $0.6380 \pm 0.0061$ & $175.35 \pm 0.92$ & $109.1 \pm 1.6$ & $1.778 \pm 0.020$ & $0.2916 \pm 0.0033$ \\
\hline & $c$ & $2448.9 \pm 5.5$ & $8559 \pm 15$ & $0.3829 \pm 0.0080$ & $268.9 \pm 1.6$ & $112.6 \pm 1.3$ & $7.997 \pm 0.095$ & $3.820 \pm 0.044$ \\
\hline HD 145934 & $b$ & $2730 \pm 100$ & $11430 \pm 370$ & $0.053_{-0.063}^{+0.053}$ & $215 \pm 62$ & $22.9 \pm 2.6$ & $2.28 \pm 0.26$ & $4.60 \pm 0.14$ \\
\hline \multirow[t]{2}{*}{ HD 183263} & $b$ & $625.10 \pm 0.34$ & $12113.0 \pm 2.4$ & $0.3728 \pm 0.0065$ & $232.9 \pm 1.4$ & $86.16 \pm 0.79$ & $3.635 \pm 0.034$ & $1.486 \pm 0.023$ \\
\hline & $c$ & $4684 \pm 71$ & $10430 \pm 310$ & $0.051 \pm 0.010$ & $299 \pm 22$ & $77.5 \pm 1.1$ & $6.90 \pm 0.12$ & $5.69 \pm 0.11$ \\
\hline \multirow[t]{2}{*}{ HD 187123} & $b$ & $3.0965886 \pm 0.0000043$ & $14342.87 \pm 0.30$ & $0.0093 \pm 0.0046$ & $360 \pm 200$ & $68.91 \pm 0.36$ & $0.5074 \pm 0.0026$ & $0.04213 \pm 0.00034$ \\
\hline & $c$ & $3324 \pm 46$ & $13625 \pm 40$ & $0.280 \pm 0.022$ & $258.5 \pm 3.9$ & $25.10 \pm 0.44$ & $1.818 \pm 0.035$ & $4.417 \pm 0.054$ \\
\hline \multirow[t]{2}{*}{ HD 217107} & $b$ & $7.126846 \pm 0.000013$ & $14395.789 \pm 0.025$ & $0.1283 \pm 0.0027$ & $24.0 \pm 1.3$ & $140.30 \pm 0.40$ & $1.4135 \pm 0.0042$ & $0.07505 \pm 0.00097$ \\
\hline & $c$ & $5189 \pm 21$ & $10770 \pm 16$ & $0.3848 \pm 0.0086$ & $206.3 \pm 1.7$ & $53.41 \pm 0.75$ & $4.513 \pm 0.072$ & $6.074 \pm 0.080$ \\
\hline \multirow[t]{2}{*}{ GJ 849} & $b^{\mathrm{c}}$ & $1924 \pm 15$ & $13770 \pm 150$ & $0.038 \pm 0.019$ & $66 \pm 28$ & $23.96 \pm 0.94$ & $0.911 \pm 0.036$ & $2.39 \pm 0.082$ \\
\hline & $c^{\mathrm{c}}$ & $5520 \pm 390^{d}$ & $14320 \pm 690$ & $0.087 \pm 0.056$ & $172 \pm 50$ & $17.5 \pm 1.1$ & $0.944 \pm 0.070$ & $4.82 \pm 0.21$ \\
\hline
\end{tabular}

Notes.

a The fit includes a linear trend of $1.8 \pm 0.4 \mathrm{~m} \mathrm{~s}^{-1} \mathrm{yr}^{-1}$.

b The fit includes a linear trend of $-3.4 \pm 0.2 \mathrm{~m} \mathrm{~s}^{-1} \mathrm{yr}^{-1}$.

${ }^{c}$ Except where noted, these parameter uncertainties were computed using only the stable bootstrapping realizations.

${ }^{\mathrm{d}}$ This parameter uncertainty was computed using the $\chi^{2}$ map.

systems where planet-planet interactions are negligible given the precision and the span of the observations. In this package, RV curves are described by both non-linear and linear parameters, and the package performs least-squares fitting on them separately. The package uses a simple linear least-squares solution for the linear parameters, and the Levenberg-Marquardt algorithm for the nonlinear parameters. RVLIN supplies a sum-of-Keplerians model (plus optional secular trend and offsets between instruments) to MPFIT, the IDL implementation of the LM method developed by Markwardt (2009).

We fit for these new planetary system as follows: (1) we collect Keck RVs before and after the 2004 HIRES upgrade separately to account for any (small) offsets between the preand post-upgrade time series (e.g., Kane et al. 2014); (2) we collect literature RVs for the system from other telescopes, if available, (3) we use the published orbital parameters (which we collect from the Exoplanet Orbit Database; Han et al. 2014) as initial guesses for the planets' orbits; (3) we use RVLIN to fit the system anew (with additional planets contributing five model parameters each, if necessary); (4) we use the reduced $\chi^{2}\left(\chi_{v}^{2}\right)$ to describe the goodness of fit.

We calculate most orbital parameter uncertainties using BOOTTRAN (Wang et al. 2012), which uses RVLIN and a bootstrapping method to compute the distribution of parameters consistent with the data. Because uncertainties can be highly non-Gaussian for planets with incomplete orbits, we also examine the minimum $\chi^{2}$ surface in minimum mass-period space (Section 3).

For our fits, we choose values for the jitter (Wright 2005, and references therein) that yield $\chi_{v}^{2}$ values close to 1 ; usually we pick a value similar to the rms of the initial fit which does not incorporate jitter. If a star has data from several (more than three) instruments taken by multiple teams, we apply jitter on an instrument-by-instrument basis. To do so, we ran the fit with no assumed jitter, calculated for each instrument the standard deviation of the residuals, and added that value in quadrature to the velocities. After that, we rerun the fit and that yielded the best-fit parameters. We utilized an instrument-by-instrument jitter for HD 24040, HD 74156, and HD 217107. In general, we 
are confident in the relative instrumental uncertainties in the preand post-upgrade HIRES data, and we use a common jitter value for both. ${ }^{6}$ Table 2 lists the stellar parameters of host stars, and Table 3 lists the orbital parameters of the planets we discussed below. Corresponding RV plots and additional figures follow the text.

Sun-like stars are known to have magnetic cycles with periods comparable to the period of Jupiter (Baliunas et al. 1995). A persistent concern in the hunt for long-term RV signals from Jupiter analogs has been that they might be mimicked by the effects of such magnetic cycles, which could alter convective patterns such that the magnitude of the diskintegrated convective blueshift of a star might vary with the stellar cycle (Dravins 1985; Walker et al. 1995; Deming et al. 1987; Santos et al. 2010).

A common way to check that magnetic effects are not responsible for RV variations is to measure correlations between the RVs and activity indices such as $\mathrm{Ca}$ II $\mathrm{H} \& \mathrm{~K}$. Previous work by Wright et al. (2008), Santos et al. (2010), and Lovis et al. (2011) find that the observed activity-cycle-induced $\mathrm{RV}$ amplitudes are typically quite small (a few $\mathrm{m} \mathrm{s}^{-1}$ or less), although there are suggestions that a few stars may show abnormally high levels of correlation (at the level of $10-20 \mathrm{~m} \mathrm{~s}^{-1}$ ).

We have checked for activity cycles in these stars to see if they have similar periods and phases to the RV measurements. To do this, we have used the $\mathrm{Ca}$ II $\mathrm{H} \& \mathrm{~K}$ chromospheric activity measurements from Wright et al. (2004, hereafter W04), Isaacson \& Fischer (2010, hereafter IF10), and more recent measurements made using the same data stream and pipeline as the latter work. For some stars, there appear to be calibration differences between the measurements published by W04 and those made using the IF10 pipeline, necessitating a rescaling or application of an offset to one of the streams. This is most apparent in "flat activity" stars which show no variation but occasionally exhibit a large jump in activity level between the two data streams.

In six of our stars, there is no appreciable activity variation (i.e., they are "flat activity" stars, Saar et al. 1998), making it very unlikely that the large RV variations we see are due to solartype activity cycles. The seventh star, HD 183263 does show a significant cycle, however. The W04 activity levels decrease from 2002 to 2004, and the IF10 show a continued decrease starting in late 2004, which bottoms out in a minimum in 2012. The actual velocities show a minimum in 2005 and a maximum in 2012, thus exhibiting a shorter period than the actual activity cycle. The negative correlation between 2005-2012 and the very high amplitude of the RV signals are inconsistent with typical stars with RV-activity correlation seen in Wright et al. (2008) and described by Lovis et al. (2011). It is thus very unlikely that any of the long-period signals we describe in this work are due to stellar magnetic activity cycles.

\subsection{Minimum Masses from Linear Trends Alone}

In some cases, we find that a secular increase or decrease in the observed radial velocities is present (a "linear trend"),

\footnotetext{
6 The effect of jitter on the best-fit values of an orbital solution is to give more even weight to points with different measurement uncertainties; in the cases of the well-detected planets we discuss in this work, the exact value of the jitter has very little effect on these best-fit values. Because we determine most of our parameter uncertainties via bootstrapping, our uncertainties are not strongly affected by our choice of jitter, and so there is no need to find the precise jitter value that yields $\chi_{v}^{2}=1.0$.
}

which is presumably a small portion of a Keplerian signal from a massive companion, typically an outer planet, or a secondary star or brown dwarf (Crepp et al. 2012, 2013a, 2013b, 2014; Montet et al. 2014; Knutson et al. 2014).

When the trend shows no curvature and we have no $\mathrm{AO}$ imagery to put limits on the mass and angular separation of companions, we usually say very little about the companion beyond a minimum mass (and a maximum luminosity from the fact that its spectrum did not complicate the RV analysis). The scenario that gives the minimum mass to a companion generating a linear trend of a given magnitude is one that has $e \sim 0.5, \omega=90^{\circ}$, which produces a sawtooth-like RV curve with a long, nearly linear component for $\sim 80 \%$ of the orbit with a brief, high-acceleration component during periastron for the other $\sim 20 \%$ (Wright 2006, and see top panel of Figure 1 for an example; other panels show other pathological cases with radically different periods and semiamplitudes that mimic the same trend).

The minimum mass of a planetary companion detected only by its strongly detected constant acceleration $\dot{\gamma}$, is thus derived by solving the mass function for the minimum mass (e.g., Wright $\&$ Howard 2009) assuming $P \sim 1.25 \tau$ (where $\tau$ is the span of the observations), $e \sim 0.5$, and $K \sim \tau \dot{\gamma}$ :

$$
M_{\text {minimum }} \approx\left(0.0164 M_{\mathrm{Jup}}\right)\left(\frac{\tau}{\mathrm{yr}}\right)^{4 / 3}\left|\frac{\dot{\gamma}}{\mathrm{m} \mathrm{s}^{-1} \mathrm{yr}^{-1}}\right|\left(\frac{M_{*}}{M_{\odot}}\right)^{2 / 3}
$$

\section{REFINED ORBITAL PARAMETERS FOR SEVEN PLANETARY SYSTEMS}

Our sample includes many known planetary systems of interest because of the presence of a linear trend in the residuals indicative of an additional companion; some with known trends with significant curvature; and some known to have outer companions with poorly constrained parameters. The first six, HD 24040, HD 66428, HD 74156, HD 183263, HD 187123, and HD 217107 are G stars; the seventh GJ 849, is an M dwarf.

Table 1 presents the time span of sets of observations, the number of points from each set, the number of new points, the mean uncertainty in velocities from each set, and the offsets between instruments.

\subsection{HD 24040}

Wright et al. (2007) reported a substellar companion to the star HD 24040 with a wide range of possible periods (10 yr $<P<$ $100 \mathrm{yr})$ and minimum masses $\left(5<M \sin i<20 M_{\text {Jup }}\right)$. Boisse et al. (2012), combining velocities from HIRES, SOPHIE, and ELODIE, determined an orbit of $3668_{-171}^{+169}$ days (corresponding to $10 \mathrm{yr}$ ) and a minimum mass of $4.01 \pm 0.49 M_{\mathrm{Jup}}$ for HD $24040 b$. Boisse et al. (2012) also found a linear trend of $3.85_{-1.29}^{+1.43} \mathrm{~m} \mathrm{~s}^{-1} \mathrm{yr}^{-1}$, indicative of a third body in the system. Boisse et al. (2012) also investigated potential longterm correlation between SOPHIE measurements and stellar activity indices but did not find such behavior.

We present an updated fit with more recent Keck-HIRES velocities, seen in Figure 2 and Table 4. We use HIRES data and published SOPHIE and ELODIE data, so in our fit we applied jitter instrument-by-instrument. With 107 velocities in total, 47 of which are from HIRES, 13 from SOPHIE, and from 47 ELODIE (Boisse et al. 2012), we find for the best-fit oneplanet Keplerian model an rms of $13.62 \mathrm{~m} \mathrm{~s}^{-1}$ and $\chi_{v}^{2}$ of 0.93 . HD $24040 b$ orbits at a semimajor axis of $4.637 \pm 0.067 \mathrm{AU}$, 

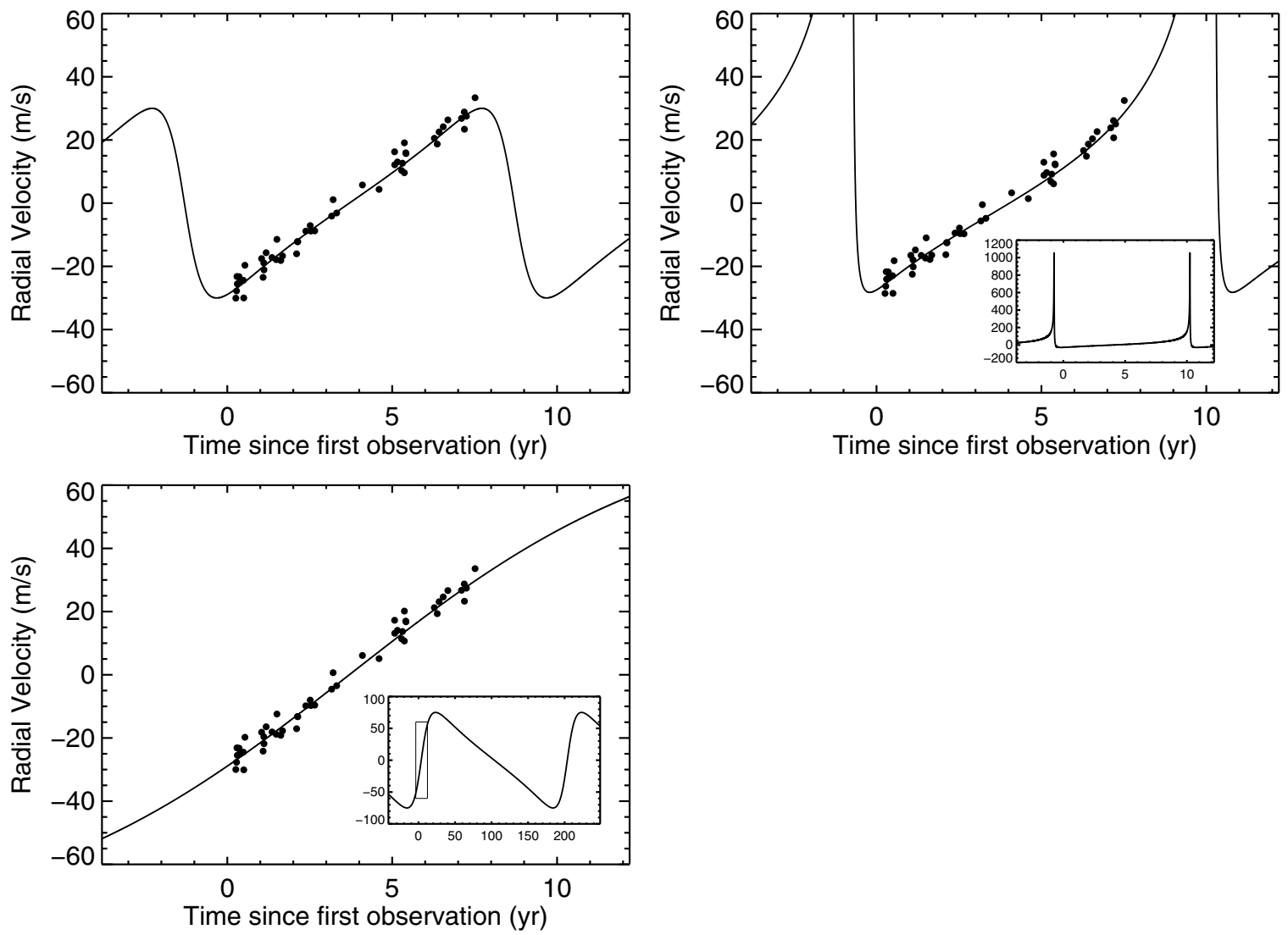

Figure 1. Fifty synthetic RV measurements made over 8 yr by an unlucky observer of a hypothetical system with Gaussian errors of $3 \mathrm{~m} \mathrm{~s}^{-1}$. Top: RV curve of a planet with $M \sin i=1.97 M_{\mathrm{Jup}}, P=10 \mathrm{yr}, K=30 \mathrm{~m} \mathrm{~s}^{-1}, e=0.5$, and $\omega=90^{\circ}$. The observer might conclude, incorrectly, that they were seeing the effects of a distant exoplanet with $P \gg 8 \mathrm{yr}$ and $K \gg 30 \mathrm{~m} \mathrm{~s}^{-1}$. Middle: RV curve of a planet with $M \sin i=6.6 M_{\mathrm{Jup}}, P=11 \mathrm{yr}, K=345 \mathrm{~m} \mathrm{~s}^{-1}, e=0.97$ and $\omega=20^{\circ}$. Although the period $P$ and magnitude of the observed trend are about the same as that in the top panel, the true semi-amplitude of the orbit is much larger. The inset illustrates the complete RV curve, with the same units as the main figure. Bottom: RV curve for hypothetical planet with $M \sin i=11.7 M_{\mathrm{Jup}}, P=200 \mathrm{yr}, K=65 \mathrm{~m} \mathrm{~s}{ }^{-1}$, $e=0.5$, and $\omega=270^{\circ}$. Although the magnitude of the observed trend is the same as that in the top and middle panels, the period in this case is much longer, while $K$ is only modestly larger. The inset illustrates the RV curve, with the same units as the main figure, over more than a complete orbit. The box in the inset illustrates the span of the main panel. All three panels are reproduced from Wright (2006).

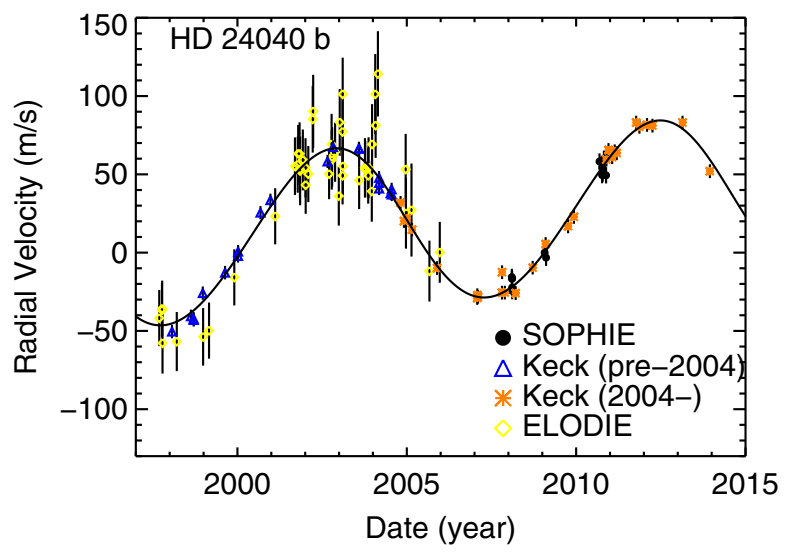

(a) HD $24040 b$

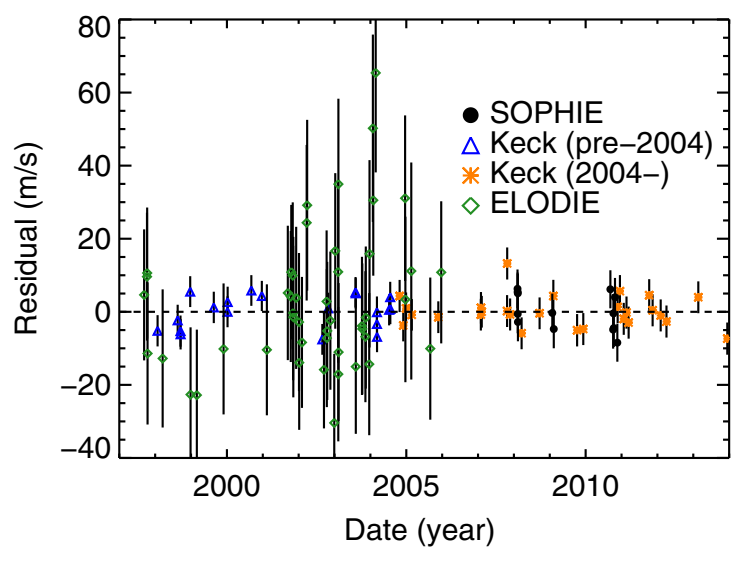

(b) Residuals

Figure 2. Radial velocity and Keplerian fit for HD $24040 \mathrm{~b}$. Solid lines represent the best-fit Keplerian orbits. The fit includes a linear trend of $1.8 \pm 0.4 \mathrm{~m} \mathrm{~s}^{-1} \mathrm{yr}^{-1}$. (a) Keck, SOPHIE, and ELODIE RVs overplotted by best-fit one-planet Keplerian model. (b) Residuals of the RVs with the best-fit one-planet Keplerian model subtracted.

corresponding to a period of $9.5 \mathrm{yr}$, making it a good Jupiter analog in terms of its orbit (however, its minimum mass is $4.10 \pm 0.12 M_{\text {Jup }}$ ). The linear trend is $1.8 \pm 0.4 \mathrm{~m} \mathrm{~s}^{-1} \mathrm{yr}^{-1}$ (lower than reported in Boisse et al. 2012), a minimum mass of at least $1.44 M_{\text {Jup }}$ according to Equation (1). Our fit for HD $24040 b$, with a period of $3490 \pm 25$ days and minimum mass of $4.10 \pm 0.12 M_{\text {Jup }}$, is in good agreement with the solution from Boisse et al. (2012).

\subsection{HD 66428}

Butler et al. (2006) announced HD $66428 b$, a planet with $P=1973 \pm 31 \mathrm{~d}(5.4 \mathrm{yr}), e=0.465 \pm 0.030$, and 


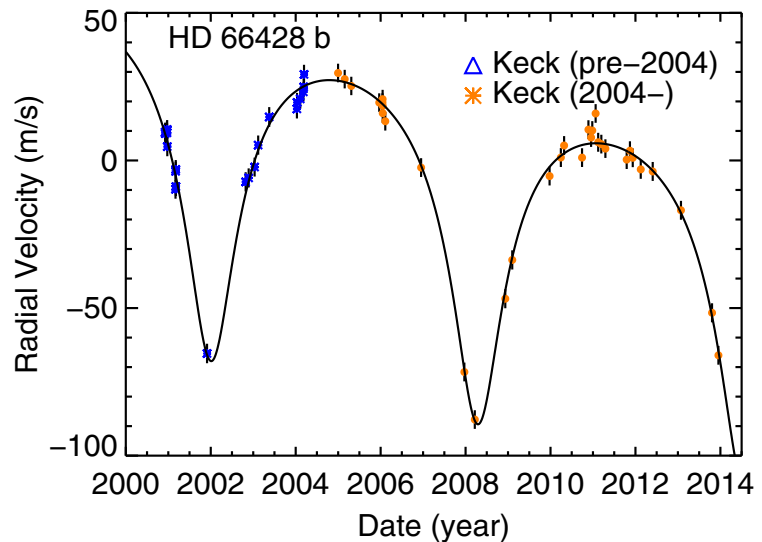

(a) HD $66428 b$

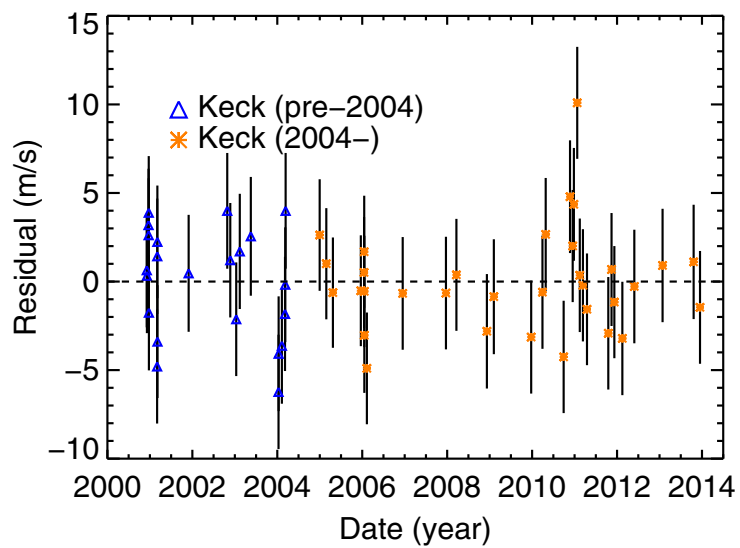

(b) Residuals

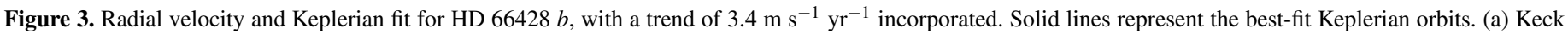
RVs overplotted by best-fit one-planet Keplerian model. (b) Residuals of the RVs with the best-fit one-planet Keplerian model subtracted.

Table 4

Radial Velocities Measured for HD 24040

\begin{tabular}{lccc}
\hline \hline $\begin{array}{l}\text { BJD } \\
-2440000\end{array}$ & $\begin{array}{c}\text { RV } \\
\left(\mathrm{m} \mathrm{s}^{-1}\right)\end{array}$ & $\begin{array}{c} \pm 1 \sigma \\
\left(\mathrm{m} \mathrm{s}^{-1}\right)\end{array}$ & Tel \\
\hline 10838.77321 & -95.2 & 1.5 & HIRES \\
11043.11965 & -85.4 & 1.5 & HIRES \\
11072.03904 & -87.0 & 1.5 & HIRES \\
11073.00232 & -87.8 & 1.1 & HIRES \\
\hline
\end{tabular}

(This table is available in its entirety in machine-readable form.)

$M \sin (i)=2.82 \pm 0.27 M_{\text {Jup }}$. We update the orbital parameters with a total of 55 velocities from HIRES (see Figure 3 ). The original fit used 29 velocities taken with HIRES from 2000 to 2006. Our new fit adds 26 new data points through late 2013. Capturing two complete orbits of HD $66428 b$, the fit has an rms of $3.14 \mathrm{~m} \mathrm{~s}^{-1}$ where we assumed a jitter of $3 \mathrm{~m} \mathrm{~s}^{-1}$ and $\chi_{v}^{2}$ of 0.96. We determine a period of $2293.9 \pm 6.4$ days, or $6.3 \mathrm{yr}$. We determine a minimum mass of $3.195 \pm 0.066 M_{\text {Jup }}$, which is more massive than reported in Butler et al. (2006).

Given our larger set of radial velocities, it is understandable that our solution does not match with the solution announced in Butler et al. (2006). The final fit finds a previously unreported linear trend of $-3.4 \pm 0.2 \mathrm{~m} \mathrm{~s}^{-1} \mathrm{yr}^{-1}$ (corresponding to a minimum mass for the outer companion of at least $1.77 M_{\text {Jup }}$, by Equation (1)).

We run the fit with no jitter and no trend in order to see the significance of the detected trend. For that case, $\chi_{v}^{2}$ is 52.56, and the rms of the residuals is $7.46 \mathrm{~m} \mathrm{~s}^{-1}$. To compare, we found an rms of $3.14 \mathrm{~m} \mathrm{~s}^{-1}$ and $\chi_{v}^{2}$ of 8.23 for seven free parameters (including the trend) and no jitter. Given the improvement in the fit with a trend included, the trend is significant. We also note that the eccentricity of the orbit is large: $0.442 \pm$ 0.016. The trend may indicate that the outer companion has influenced the orbit of the $b$ component. Further monitoring will determine the nature of the source of the trend (i.e., whether it is due to a stellar or planetary companion).

\section{3. $H D 74156$}

Naef et al. (2004) described the HD 74156 two-planet system as a $1.86 \pm 0.03 M_{\text {Jup }}$ planet in a $51.64 \pm 0.011$ day period with a $6.17 \pm 0.23 M_{\text {Jup }}$ outer companion in a $5.5 \mathrm{yr}$ orbit. Multiple authors have suspected a third planet in the system. Barnes \&
Raymond (2004) predicted one based on the Packed Planetary System hypothesis, and Bean et al. (2008) claimed the discovery of a companion with $P=336$ days as the third planet. Based on analysis of RV jitter, Baluev (2009) questioned the validity of HD 74156 " $d$ " as a false detection due to annual systematic errors from HRS. Wittenmyer et al. (2009) concluded that the third planet was unlikely to be real, and Meschiari et al. (2011) updated the system with further observations and reached the same conclusion.

Here, we combine 226 velocities from CORALIE and ELODIE (44 and 51 observations Naef et al. 2004), HRS (82 Bean et al. 2008), and HIRES (52) (see Figure 4). We apply a two-planet Keplerian model. We added jitter instrument-byinstrument, and our fit has an rms of $11.03 \mathrm{~m} \mathrm{~s}^{-1}$ and $\chi_{v}^{2}$ of 0.97. We have captured at least two orbits of HD $74156 c$, making our orbital solution more robust than previously reported solutions. Table 3 lists the orbital parameters. HD $74156 c$ is one of the more massive planets we have examined, with minimum mass $7.997 \pm 0.095 M_{\text {Jup }}$. Both planets have large orbital eccentricities ( $e=0.64$ and $e=0.38$ for $b$ and $c$ respectively).

In Figure 5 we plot the Lomb-Scargle periodogram (Scargle 1982; Horne \& Baliunas 1986) of the residuals to our best twoplanet fit. There is no indication of any power at the period of the purported $d$ component, a result which is consistent with prior refutations of this signal (indeed, our analysis here uses much of the same data as previous work on the topic). Indeed, there is no hint of significant power at any period, indicating that there is no detectable third planetary companion in this system.

\subsection{HD 183263}

First reported by Marcy et al. (2005), the HD 183263 system showed a residual linear trend in addition to a $3.7 M_{\text {Jup }}$ planet in a 634-day period. Wright et al. (2007) attributed the new and significant curvature in the residuals to an outer companion. Wright et al. (2009) followed up and constrained the minimum mass $\left(3.57 \pm 0.55 M_{\text {Jup }}\right)$ and period $(8.4 \pm 0.3 \mathrm{yr})$ for the outer companion, HD $183263 c$, to which we report an updated set of parameters.

With 66 velocities from HIRES, we implemented a fit with an rms of $3.68 \mathrm{~m} \mathrm{~s}^{-1}$ and an assumed jitter of $3.2 \mathrm{~m} \mathrm{~s}^{-1}$. Figure 6 presents the RV curves for the system as well as the residuals. The orbit for HD $183263 c$ appears to have finally closed, and it is significantly closer to circular $(e=0.051 \pm 0.010)$ and has a longer period than the solution from Wright et al. (2009), 


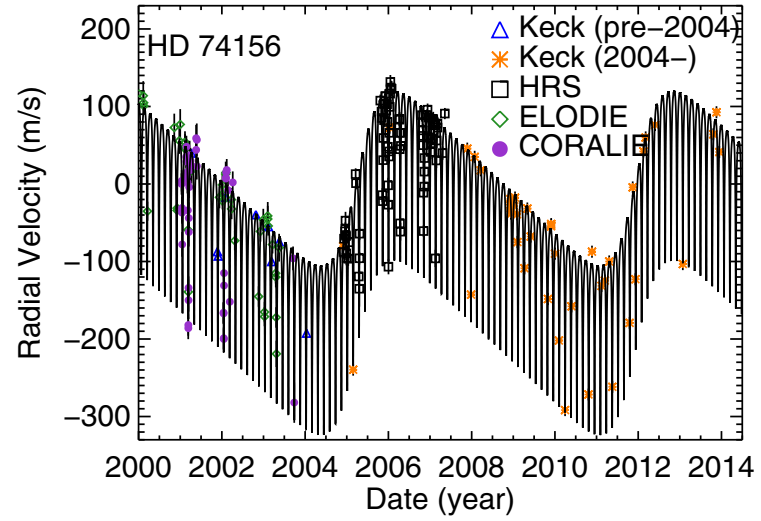

(a) HD 74156 system

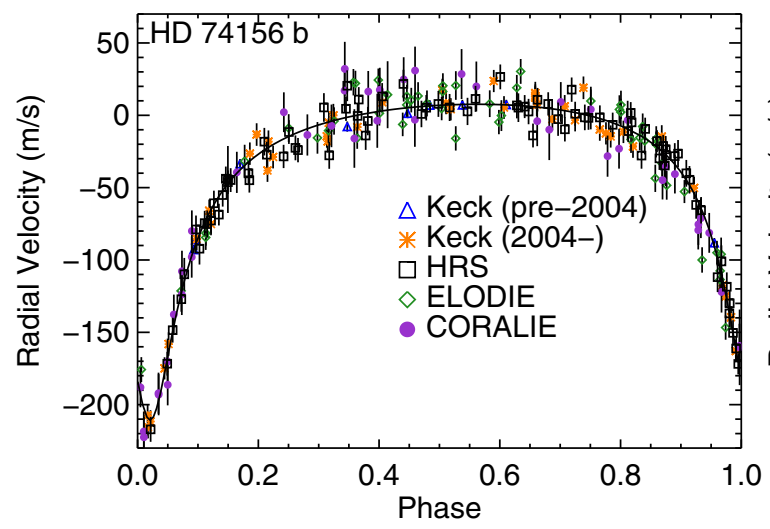

(c) HD 74156 b

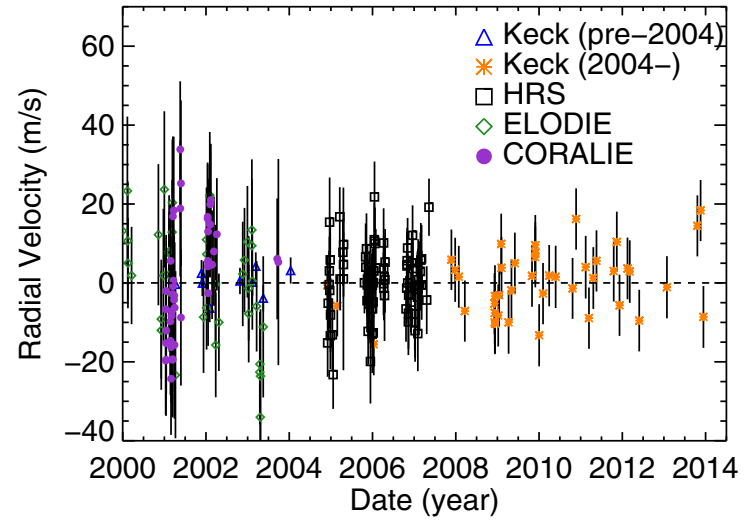

(b) Residuals

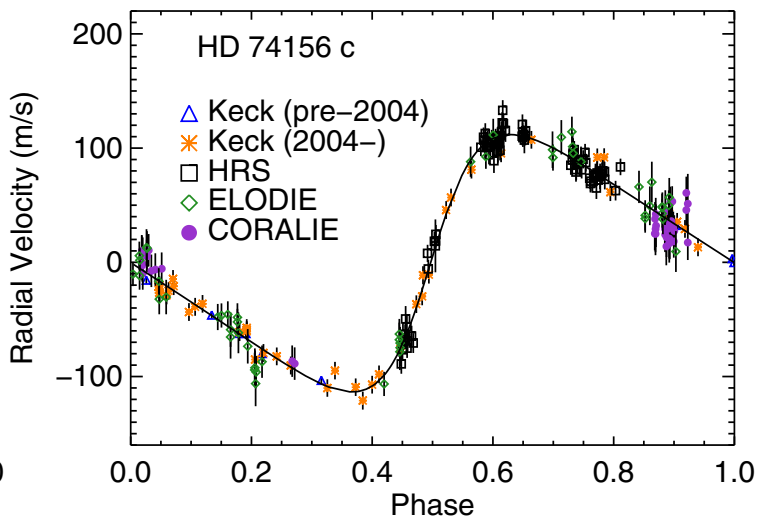

(d) HD $74156 c$

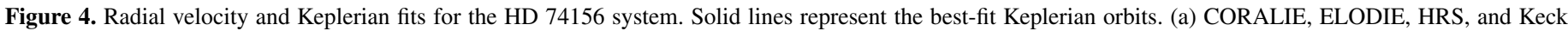

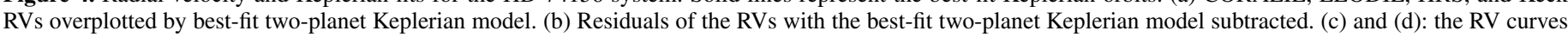
for HD $74156 b$ and $c$, respectively.

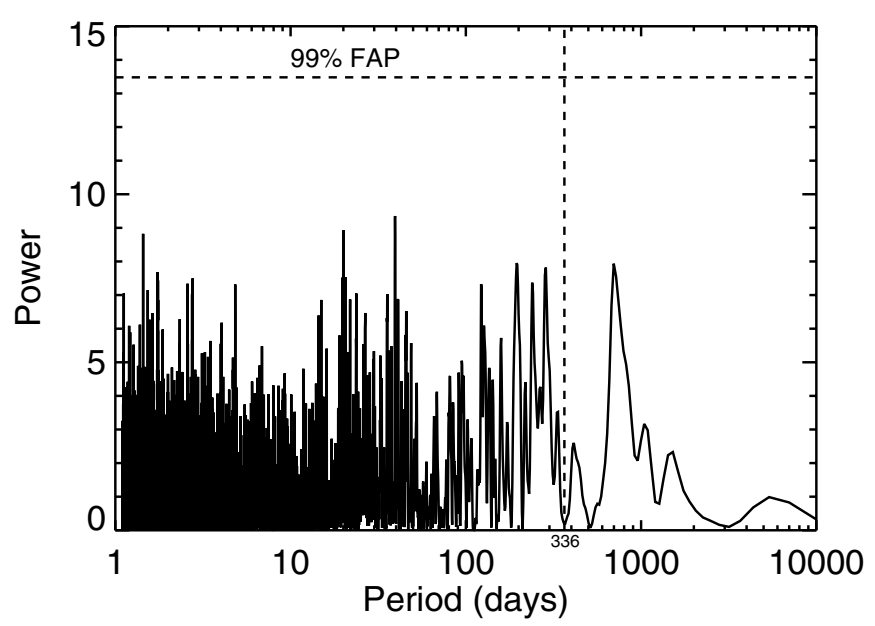

Figure 5. Periodogram of the residuals to our best two-planet, five-instrument fit to the RV data for HD 74156. There is no indication of significant power at any period, or of any power at all at 336 days, the period of the purported but disproven $d$ component. We have computed the $99 \%$ false alarm probability in this figure by calculating the highest peak in each of 10,000 such periodograms calculated for synthetic data sets of RV residuals (e.g., Howard et al. 2009). We calculated each of the 10,000 synthetic sets by randomly assigning the actual residuals (drawn with replacement) to each of the times of observations of the actual observations. In $99 \%$ of cases the tallest peak had power below 13.5.

which found $e=0.239 \pm 0.64$ and $P \sim 8.5 \mathrm{yr}$. We find for HD $183263 c$, that $P=4684 \pm 71$ days, or $9.1 \mathrm{yr} ; M \sin i$ is $6.90 \pm 0.12 M_{\text {Jup }}$. While our best-fit orbital solution does not match well with the previous orbital solution, our solution resides comfortably within the stable portion in the $P_{c}-M_{c} \sin i_{c}$ space found by Wright et al. (2009, see their Figure 3).

\section{5. $H D 187123$}

Butler et al. (1998) discovered HD $187123 b$, a $0.52 M_{\text {Jup }}$ planet in a 3 day orbit. After many years of continued monitoring of this system, Wright et al. (2007) announced a long-period outer companion with $P>10 \mathrm{yr}$ and a minimum mass between $1.5 M_{\text {Jup }}$ and $10 M_{\text {Jup }}$. Wright et al. (2009) presented a solution that constrained the mass and period of an outer companion to within $20 \%$, with $P=10.4 \pm 1.2 \mathrm{yr}$ and $M \sin i=2.0 \pm 0.3 M_{\text {Jup }}$. Figure 7 shows an updated fit with HIRES data. Naef et al. (2004) provide ELODIE velocities; however, since they have significantly worse precision and do not add temporal coverage, we do not use them here. The 108 Keck observations still cover multiple orbits of the planets; assuming a jitter of $2.23 \mathrm{~m} \mathrm{~s}^{-1}$, we find an rms of $2.66 \mathrm{~m} \mathrm{~s}^{-1}$. From our fit, the period of HD $187123 c$ is $9.1 \pm 0.13 \mathrm{yr}$ and the minimum mass is $1.818 \pm 0.035 M_{\text {Jup }}$. HD $187123 c$ appears to be a Jupiter analog, although its orbit is somewhat eccentric at $e=0.280 \pm 0.022$.

\section{6. $H D 217107$}

Fischer et al. (1999) presented HD $217107 b$ as a $1.27 M_{\text {Jup }}$ planet in a 7.12 day period. A few years later, Fischer et al. (2001) identified a linear trend in the residuals, which was likely caused by an outer companion. Vogt et al. (2005) reported the first orbit for HD $217107 c$, modestly constrained at $P=8.6 \mathrm{yr}$ 


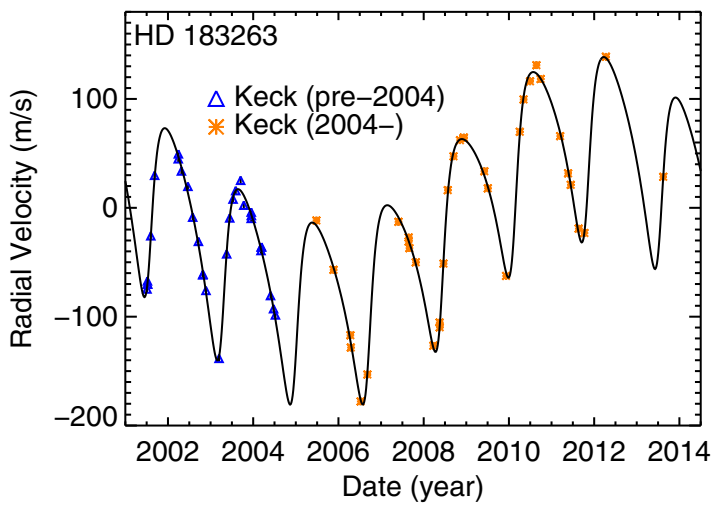

(a) HD 183263 system

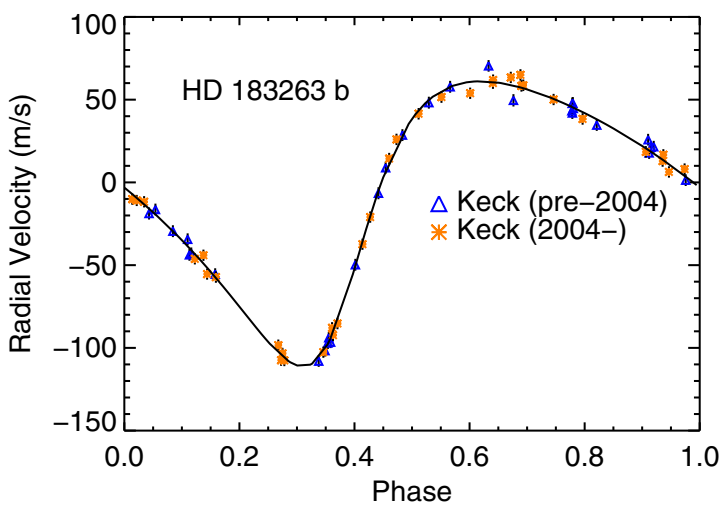

(c) HD $183263 b$

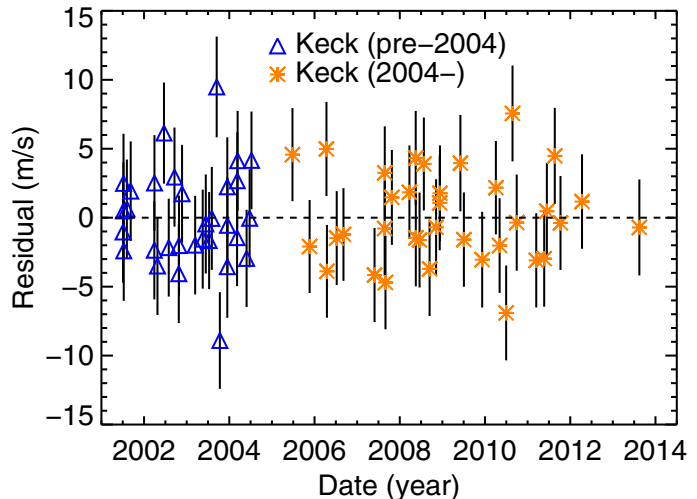

(b) Residuals

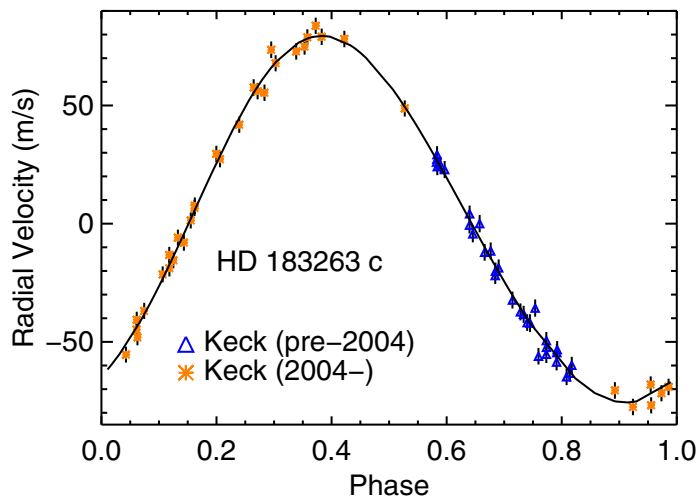

(d) HD $183263 c$

Figure 6. Radial velocity and Keplerian fits for the HD 183263 system. Solid lines represent the best-fit Keplerian orbits. (a) Keck RVs overplotted by best-fit two-planet Keplerian model. (b) Residuals of the RVs with the best-fit two-planet Keplerian model subtracted. (c) and (d): the RV curves for HD $183263 b$ and $c$, respectively.

and $M \sin i=2.1 M_{\mathrm{Jup}}$. Wright et al. (2009) constrained the orbit and mass of HD $217107 c$ to almost within $10 \%$, with $P \sim 11.7 \mathrm{yr}$ and the minimum mass $\sim 2.6 M_{\text {Jup }}$.

As with the case of HD 74156, we also have data taken by different teams from several instruments, we added jitter instrument-by-instrument. In our fit, we use velocities from Keck (128 observations), Lick (Wright et al. 2009, 121), and CORALIE (63 Naef et al. 2001) to find a fit an rms of $10.29 \mathrm{~m} \mathrm{~s}^{-1}$ (see Figure 8).

Because the outer planet has only barely (apparently) completed an orbit, its orbital parameters may be especially uncertain (and are particularly sensitive to the assumption that there is not a third, longer-period planet contributing significantly to the velocities). To explore the robustness of our derived orbital period of the $c$ component as a function of its minimum mass, we have constructed a $\chi^{2}$ map in $P-M \sin i$ space (a variety of what Knutson et al. 2014 call "Wright diagrams"; see Patel et al. 2007, Wright et al. 2009 and similar approaches taken in, e.g., Dumusque et al. 2011; Boisse et al. 2012). In this map all orbital parameters have been optimized (i.e., they are at their maximum likelihood in a $\chi^{2}$ minimum sense) for each pair of $P_{c}$ and $M_{c} \sin i_{c}$ in the map (except for the offsets among the four instruments, which are held constant at their overall best-fit values).

Figure 9 shows the $\chi^{2}$ contour map, revealing that the orbital period and minimum mass for HD $217107 c$ are well constrained with $P=14.215_{-0.04}^{+0.045} \mathrm{yr}$ and $M \sin i=4.51_{-0.02}^{+0.07} M_{\text {Jup }}$. These uncertainties are roughly consistent with the uncertainties determined via bootstrapping, which yields $P=14.215 \pm$ $0.06 \mathrm{yr}$ and $M \sin i=4.51 \pm 0.07 M_{\mathrm{Jup}}$. This validates our choice of stellar jitter for this star, since the contours in the $\chi^{2}$ maps are sensitive to the choice of jitter, while the bootstrapping uncertainties are almost completely independent of it. We report the bootstrapping uncertainties in Table 3.

To test the importance of our assumption that there are only two planets contributing detectable accelerations to the star, we repeated our bootstrapping analysis with a model that includes an additional, linear trend to the data. Though there is no statistical need to include such a trend in our model, giving our model the freedom to include one could, in principle, affect the best-fit parameters for the outer planet. Indeed, though the parameters of the $b$ component do not change significantly in this model (as expected given its high frequency), three parameters for the $c$ component do change $\left(P_{c}=5120 \pm 85 \mathrm{~d}\right.$, $T_{\mathrm{p}, c}=10820 \pm 50(\mathrm{JD}-2440000)$, and $\left.K_{c}=140.7 \pm 0.5\right)$, resulting in a minimum mass for the outer companion of $4.37 M_{\text {Jup }} \pm 0.26$. The uncertainties on the parameters of the $c$ component in the with-trend model are larger by a factor of 2-4, comfortably including the parameter estimates from the no-trend model. We conclude that our choice not to include a linear trend does not have a large effect on our conclusions or parameter estimations.

\subsection{GJ 849}

\subsubsection{Orbital Fit}

Unlike the other stars in this work, GJ 849 is an M3.5 dwarf. Various studies of this star's composition have all found similar, super-solar abundances: Rojas-Ayala et al. (2012) find $[\mathrm{Fe} / \mathrm{H}]=$ $0.31 \pm 0.17$ (from $K$-band features); Önehag et al. (2012) find 


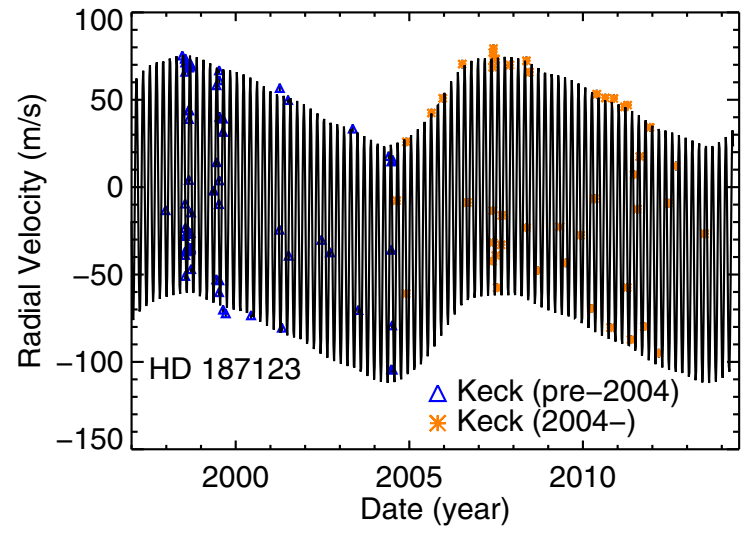

(a) HD 187123 system

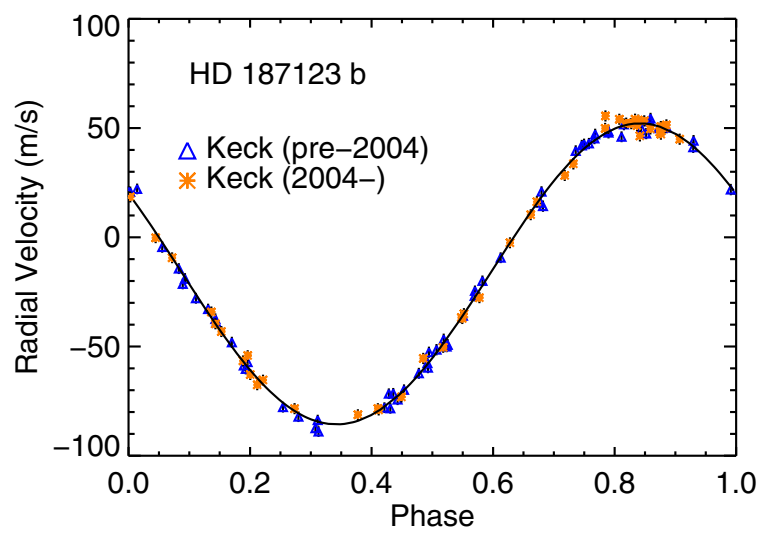

(c) HD $187123 b$

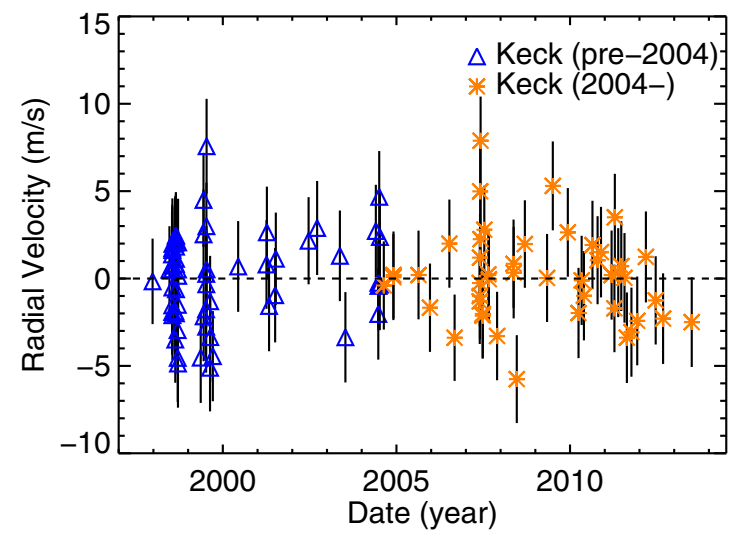

(b) Residuals

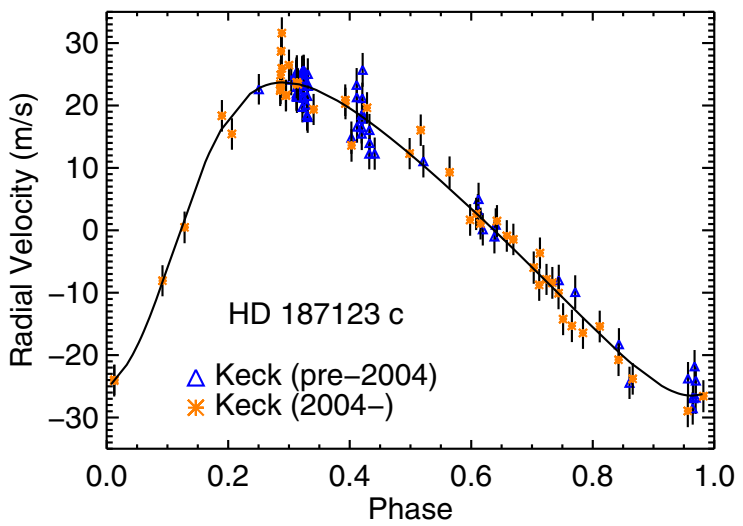

(d) HD $187123 c$

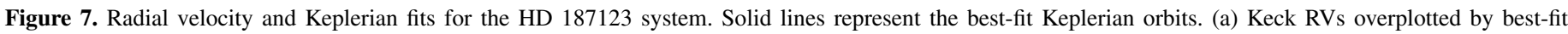

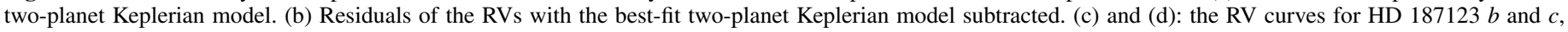
respectively.

$0.35 \pm 0.10$ (using $J$-band); and Terrien et al. (2012) found $0.31 \pm 0.12$ (using $K$-band).

GJ 849 hosts the first planet discovered orbiting an M-dwarf with a semi-major axis greater than 0.21 AU. Butler et al. (2006) announced GJ $849 b$, with $P=5.16 \mathrm{yr}$ and minimum mass $0.82 M_{\text {Jup }}$. At the time, there was evidence of a linear trend of $-4.75 \mathrm{~m} \mathrm{~s}^{-1} \mathrm{yr}^{-1}$, indicative of a second companion. Bonfils et al. (2013) also fitted the system with one planet and a linear trend of $-4.0 \mathrm{~m} \mathrm{~s}^{-1} \mathrm{yr}^{-1}$, adding their HARPS data to the published HIRES velocities.

Stellar magnetic activity had to be ruled out as the source of the trend. Gomes da Silva et al. (2012) monitored several M-dwarfs from the HARPS program for long-term magnetic activity. For GJ 849, they saw mild correlation in our velocities with the $\mathrm{Na}$ I index data. However, the amplitude was not large enough. Montet et al. (2014) provided the first orbital parameters for on GJ $849 c$, finding $M \sin i=0.70 \pm 0.31 M_{\mathrm{Jup}}$, and $P=19.3_{-5.9}^{+17.1} \mathrm{yr}$, and found no correlation between stellar magnetic activity and the long-period signal of this outer companion.

Our fit, using 35 velocities from HARPS (Bonfils et al. 2013) and 82 velocities from HIRES spanning from 1997 through early 2014, has further constrained the orbital parameters of the GJ 849 system. We incorporate a jitter of $3 \mathrm{~m} \mathrm{~s}^{-1}$, and our fit has an rms of $3.72 \mathrm{~m} \mathrm{~s}^{-1}$.

GJ $849 b$ is a $0.911 M_{\text {Jup }}$ planet in a $5.27 \mathrm{yr}$ period with an orbital eccentricity of 0.038 . GJ $849 c$ is a $0.944 \pm 0.07 M_{\text {Jup }}$ planet in a $15.1 \pm 0.66 \mathrm{yr}$ period with an orbital eccentricity of $0.087 \pm 0.06$.

GJ $849 c$ has the longest robustly measured orbital semimajor axis of any planet orbiting an $\mathbf{M}$ dwarf discovered to date. Indeed, it has one of the longest well-measured periods of exoplanets orbiting any kind of star. Exoplanets with similar periods and period uncertainties in the literature include $55 \mathrm{Cnc}$ $d$ (Marcy et al. 2002; Endl et al. 2012); HD $166724 b$ and HD $219077 b$ (Marmier et al. 2013); and HD 13931 $b$ (Howard et al. 2010) — but these all orbit stars with $M>0.8 M_{\odot}$ and the two from Marmier et al. show significant eccentricity. The exoplanet with the longest robustly measured orbital period is $\beta$ Pictoris $\left(P=20.5_{-1.4}^{+2.9}\right.$ yr Macintosh et al. 2014).

We estimated the model parameters for GJ 849 in two additional ways to check for consistency and robustness. It is unclear whether the bootstrap resampling procedure provides an accurate estimate of GJ 849 c's orbital parameters. In particular, the poor phase coverage before 2001 (see Figure 10) results in several clear outlier models in the joint parameter distributions.

In our first check for accuracy in the parameters and uncertainties, as with HD $217107 c$, we constructed a $P-M \sin i$ $\chi^{2}$ map to confirm that the orbital period of GJ $849 c$ is well constrained (assuming no additional planets and a stellar jitter of $3 \mathrm{~m} \mathrm{~s}^{-1}$ ), despite having just completed an orbit, and find that the $68 \%$ confidence interval contours corresponds to uncertainties in $P$ of less than 5\%. As Figure 11 shows, the $\chi^{2}$ map uncertainties in minimum mass are $0.07 M_{\text {Jup }}$, exactly consistent 


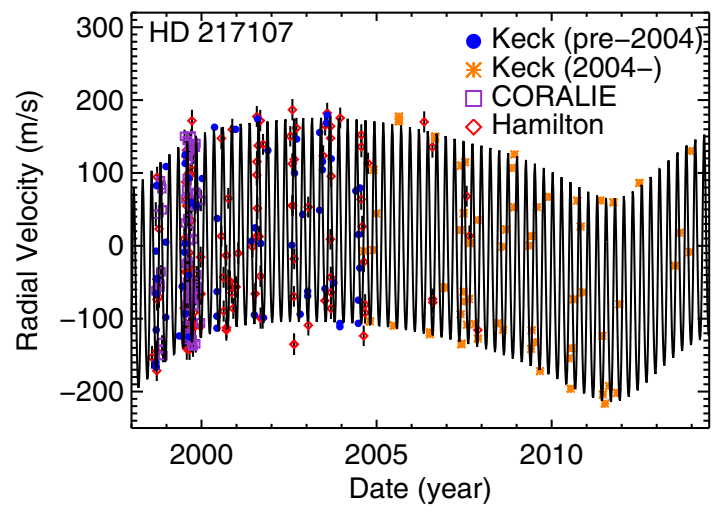

(a) HD 217107 system

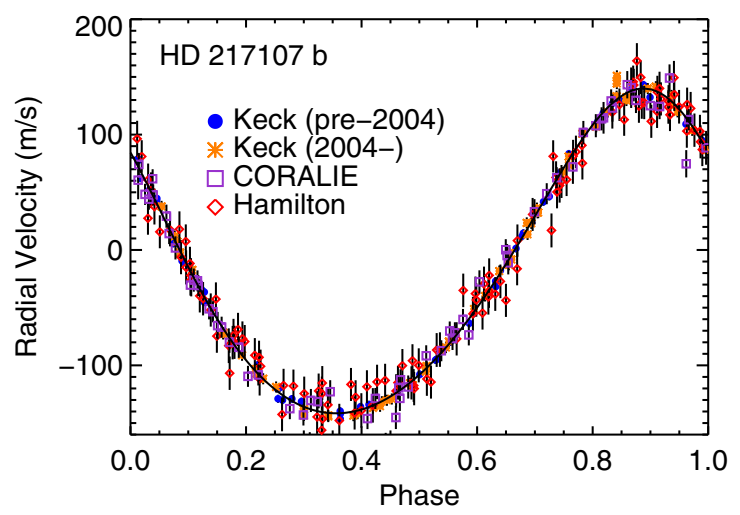

(c) HD $217107 b$

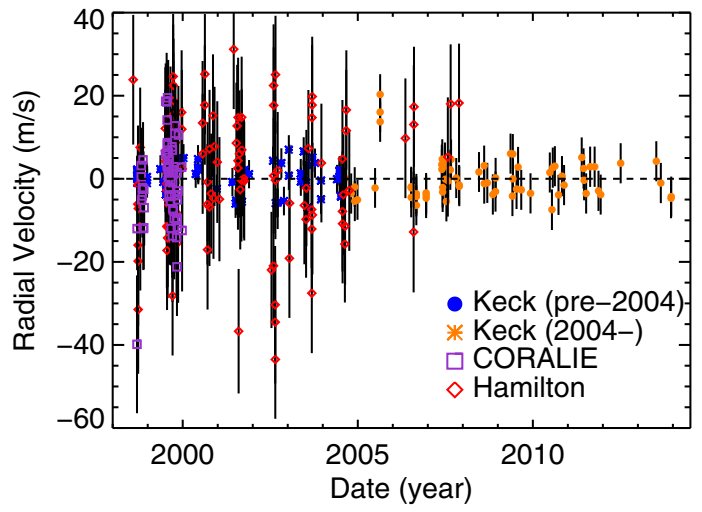

(b) Residuals

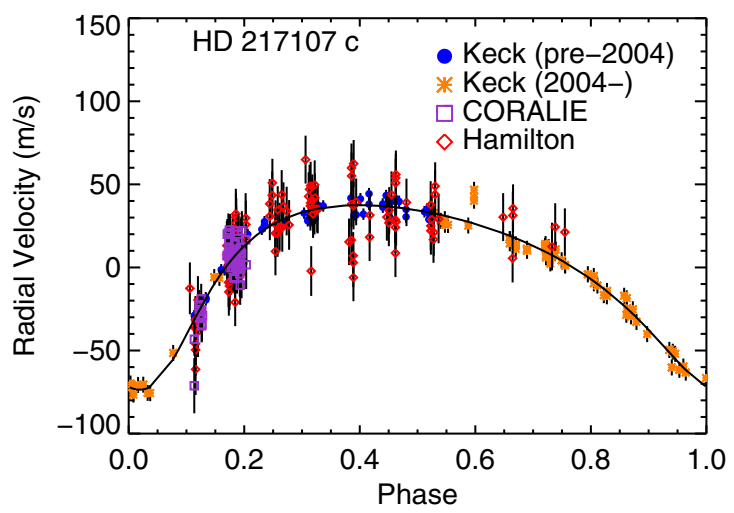

(d) HD $217107 c$

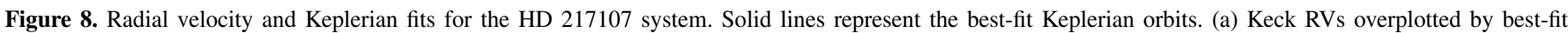

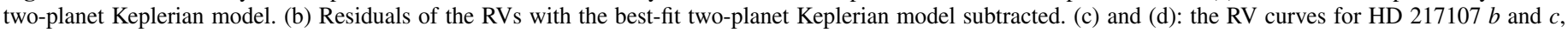
respectively.

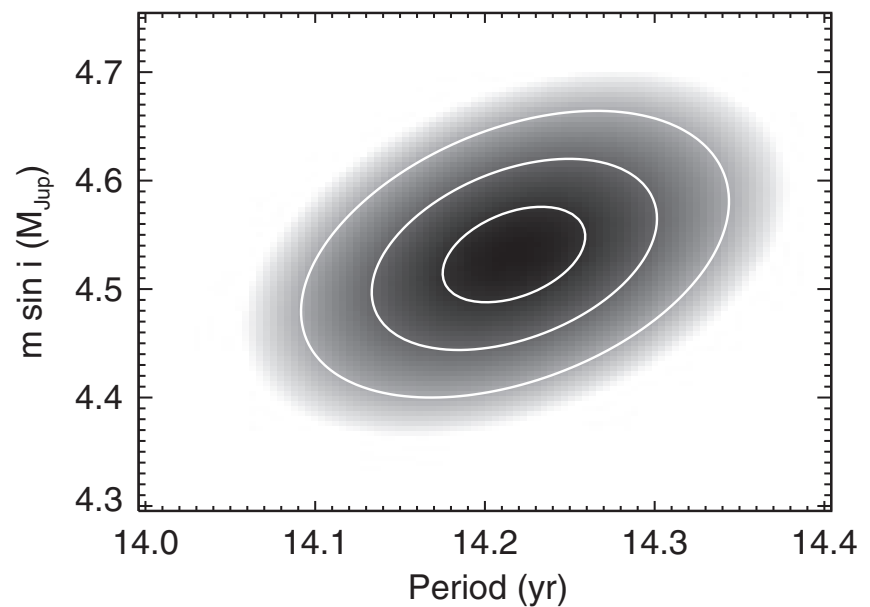

Figure 9. Best-fit $100 \times 100 \chi^{2}$ map for fixed values of $P_{c}$ and $M_{c} \sin i_{c}$ for HD $217107 c$. This confirms that the period and mass are well-constrained. We have illustrated the contours of the $1 \sigma, 2 \sigma$, and $3 \sigma$ (defined by $\chi^{2}=$ $\left.\chi_{\min }^{2}+\{2.30,6.17,11.8\}\right)$ confidence levels, based on for the number of degrees of freedom in the problem (Press et al. 2002). The center and $1 \sigma$ limits in both parameters are consistent with the bootstrapping uncertainties for these parameters.

with our bootstrapping errors; the uncertainties in period are $\sim 1.1 \mathrm{yr}$, which is larger than the bootstrapping errors of $0.66 \mathrm{yr}$, probably because the $\chi^{2}$ contours are asymmetric.

As a second check, we turn to a Bayesian approach for performing parameter estimation via Markov chain Monte Carlo. We adopt the usual broad priors for Keplerian orbital parameters and likelihood assuming uncorrelated, Gaussian measurement errors with dispersion based on the quadrature sum of the reported measurement uncertainties and an unknown jitter term (Ford 2006). Given the potential for mutual planetary interactions, we apply RUN DMC, ${ }^{7}$ a well tested code that combines n-body integration with differential evolution Markov chain Monte Carlo (Nelson et al. 2014). Although the GJ 849 planets are well approximated by Keplerian orbits, the differential evolution proposal in RUN DMC is much more efficient than a traditional random walk MCMC for dealing with correlated parameters, which are often present in the parameters for longperiod companions, and so by using RUN DMC we do not have to fine tune a proposal distribution. We find that the marginal posterior probability distribution for $P_{c}$ has $68 \%$ of its mass within $0.74 \mathrm{yr}$ of the median period of $15.1 \mathrm{yr}$, only slightly larger than the uncertainty estimated from the bootstrap.

The similarity of the parameter uncertainties from all three methods verifies that the orbit of GJ $849 c$ is well constrained and validates the BOOTTRAN and $\chi^{2}$ map approaches (in this case) and our choice of jitter. We use the more conservative $\chi^{2}$ contours do determine parameter uncertainties in Table 3.

\subsubsection{Stability}

Because this system is not "highly hierarchical" (Wright 2010) in mass or orbital period, we have performed $n$-body simulations to establish the dynamical stability of our orbital

\footnotetext{
7 We used the Keplerian parameter priors given in Nelson et al. (2014), and the algorithmic parameters $n_{\text {chains }}=300, n_{\text {gen }}=100,000, \sigma_{\gamma}=0.01$, and MassScaleFactor $=1.0$
} 


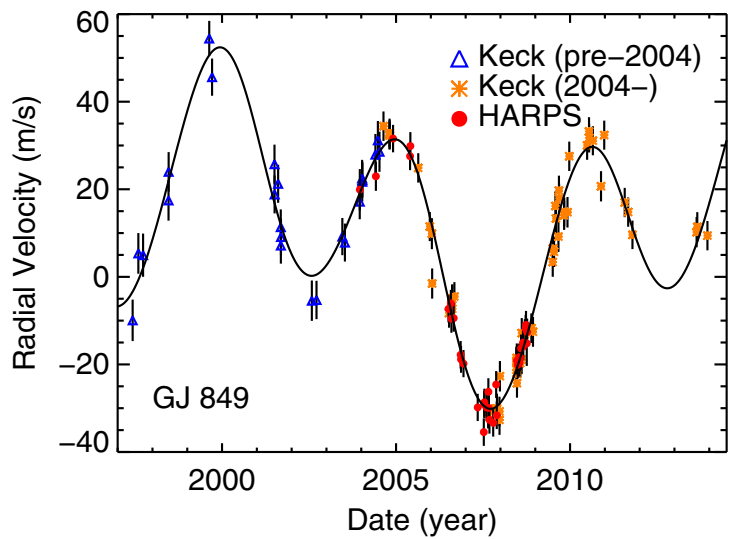

(a) GJ 849 system

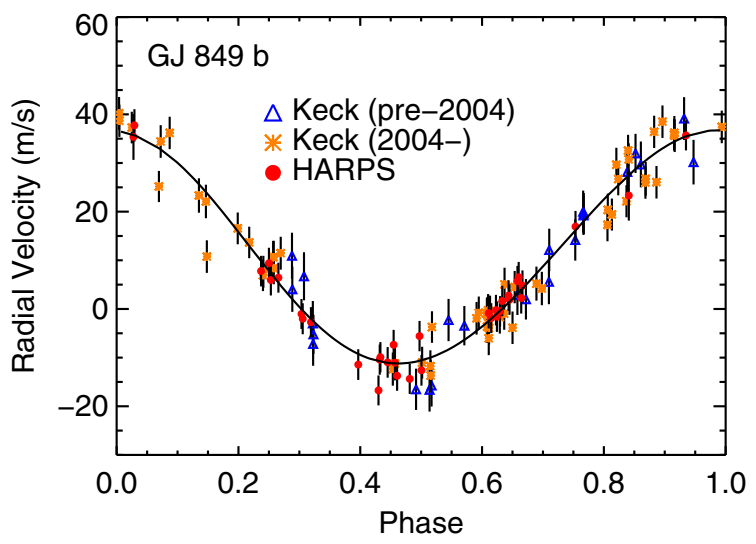

(c) GJ $849 b$

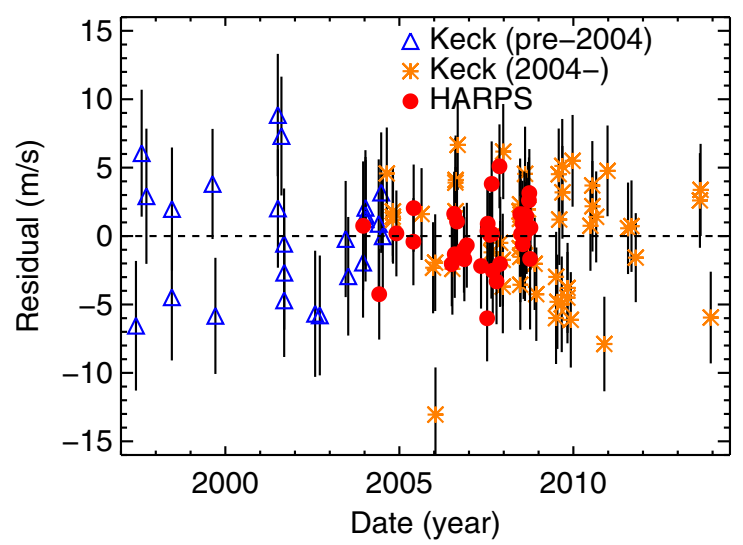

(b) Residuals

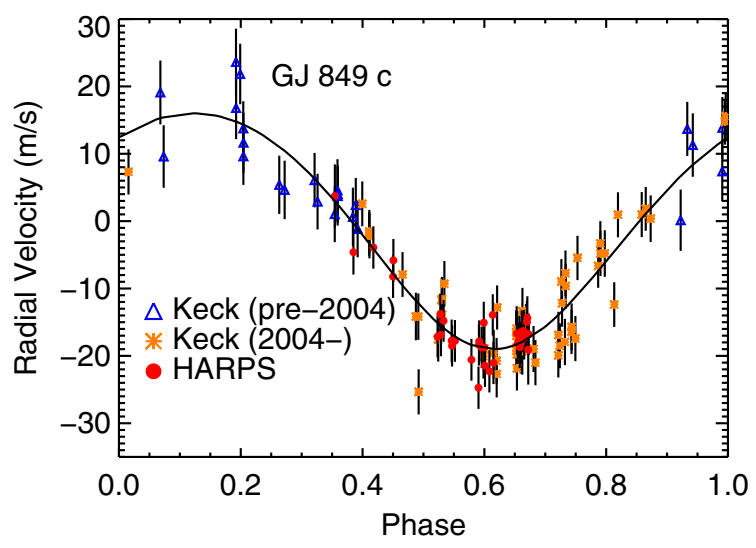

(d) GJ $849 c$

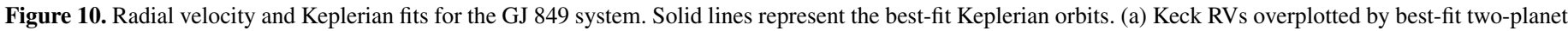
Keplerian model. (b) Residuals of the RVs with the best-fit two-planet Keplerian model subtracted. (c) and (d): the RV curves for GJ $849 b$ and $c$, respectively.

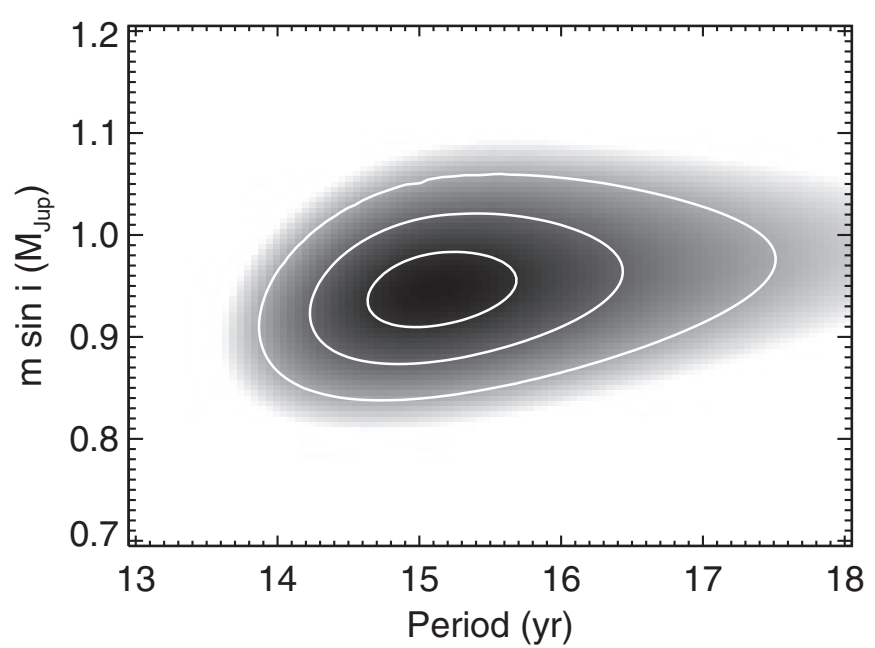

Figure 11. Contours at $1 \sigma, 2 \sigma$, and $3 \sigma$ (defined by $\chi^{2}=\chi_{\min }^{2}+$ $\{2.30,6.17,11.8\})$ confidence levels, as appropriate for the number of degrees of freedom in the problem (Press et al. 2002) for the orbital parameters of GJ $849 c$. The period and mass of the $c$ component appear to be well constrained it better than $5 \%$.

solutions. The 1000 BOOTTRAN realizations of the GJ 849 RV data are used to determine parameter uncertainties is associated with a complete set of Keplerian orbital parameters for the two planets $\left(P, e, \omega, K\right.$, and $T_{\mathrm{p}}$ for each planet, plus an overall $\mathrm{RV}$ offset $\gamma$ and two offsets among the three RV data sets). All of these realizations returned reasonable fits, indicating that the fitting procedure did not fail in any case.

We performed long-term dynamical integrations for all 1000 fits to these realizations of the data using the MERCURY symplectic integrator (Chambers 1999). Each simulation runs for $10^{7}$ orbits of the inner-most planet $\left(\sim 2 \times 10^{10}\right.$ days $)$. This integration timescale is short relative to the lifetime of the star but sufficiently long enough to show a significant fraction of our models undergo an instability, described below.

An instability occurs if at any point during the integration either planet crosses the other's Hill sphere or either of the planets' semi-major axes change by more than $50 \%$ of their initial value.

None of our models resulted in a collision over the course of the integration. However, we find 67 models undergo the second listed mode of instability ( $\left.\left[a_{\text {final }}-a_{\text {initial }}\right] / a_{\text {initial }} \mid>0.5\right)$ when the periastron passage of GJ $849 c$ is less than $3.5 \mathrm{AU}$ (Figure 12). The instability times are logarithmically uniform from $\sim 10$ to $\sim 10^{7} \mathrm{yr}$.

We removed the unstable BOOTTRAN realizations from our calculations of the uncertainties in the orbital parameters we report in Table 3.

\section{A $2 M_{\text {Jup }}$ PLANET AROUND HD 145934}

We here announce a new long-period planet orbiting the giant star HD 145934, a $1.748 \pm 0.105 M_{\odot}$ star (Takeda et al. 2007). This star was not known to be a giant when the California Planet 


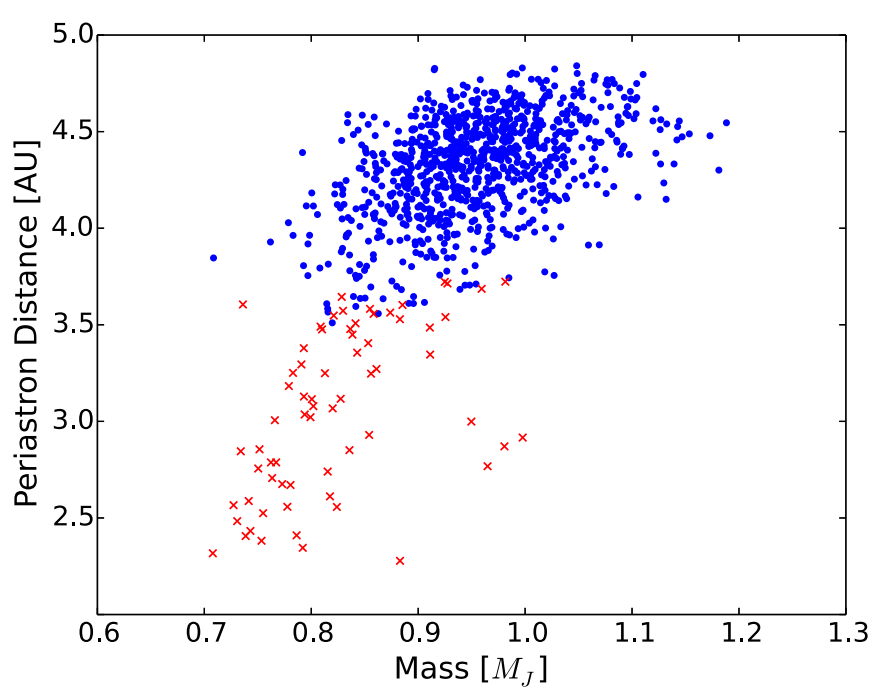

Figure 12. Best-fit minimum mass and periastron distance for all of the 1000 BOOTTRAN realizations of the GJ 849 system (which were generated through a bootstrapping procedure performed on the radial velocity data). Red x's represent fits that proved to be unstable in fewer than $10^{7}$ orbits. There is a clear boundary at periastron distance $\sim 3.6$ (AU) between stable and unstable configurations. We have not used the unstable realizations in determining parameter uncertainties for the GJ 849 system.

Survey began monitoring it in 1997 at the Keck Observatory. Since then, its $\log (\mathrm{g})$ value and mass from Jakeaa (2007) indicate that it is a grant-visual inspection of the gravity sensitive sodium and magnesium lines confirm this diagnosis. Radial

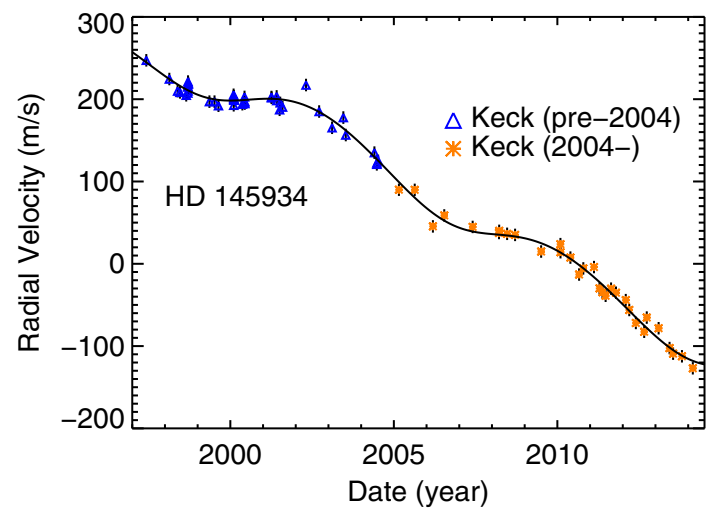

(a) HD 145934

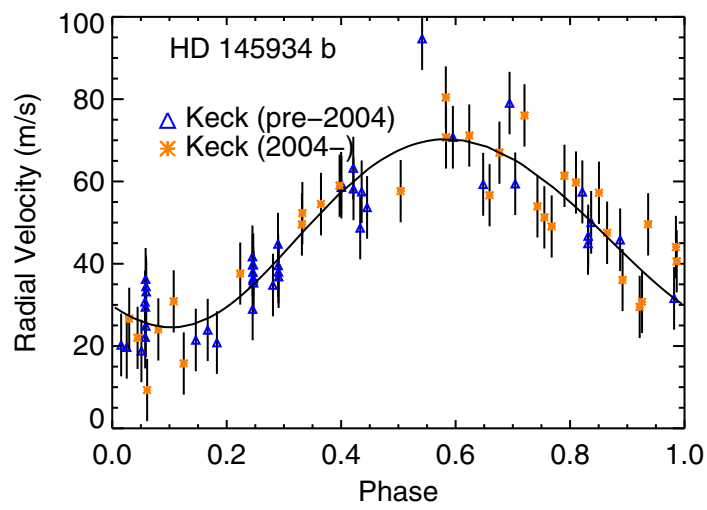

(c) HD $145934 b$ velocities of HD 145934 show a clear sinusoidal modulation of planetary amplitude upon a large linear trend, indicative of a stellar binary companion.

In our analysis of the 75 HIRES velocities for HD 145934, we note the slight overall curvature present (see Figure 13(d)). To account for the curvature using RVLIN, which (at the moment) only accommodates purely linear trends, we treated HD 145934 as a two-companion system, with the outer companion having a very long $(60 \mathrm{yr})$ orbital period and circular orbit. There is not enough information in our time series for the resulting orbital parameters of the outer companion to be meaningful, but this approach provides us sufficient flexibility to fit out the low-frequency power contributed by the binary companion. Equation (1) constrains the minimum mass of the companion to be at least $21 M_{\text {Jup }}$.

To determine the effects of modeling the ostensible stellar companion with our choice of orbital parameters on the planet's parameters, we checked first the impact of letting eccentricity be a free parameter. The best-fit eccentricity is close to circu$\operatorname{lar}(0.05)$, so our choice of fixed $e=0$ is not strongly affecting our analysis. We also changed the (fixed) period of the stellar companion to take values between 50 and $80 \mathrm{yr}$ (guesses outside the range returned poor fits, but given the nonlinear nature of the problem this does not necessarily reflect an actual upper limit to the companion's period). We found that the choice of period did not have significant impact on the parameters of the planet. For example, the best-fit values for the period of HD 145934 $b$ varied on the order of 10 days for different outer companion periods. The minimum mass varied on the order of $0.1 M_{\mathrm{Jup}}$.

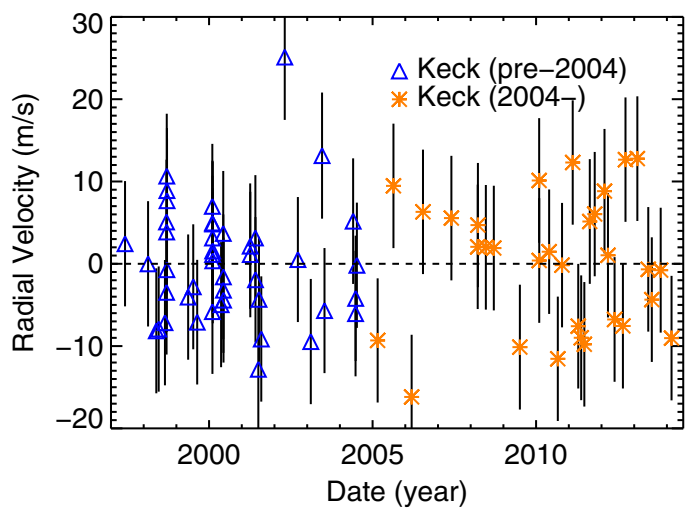

(b) Residuals

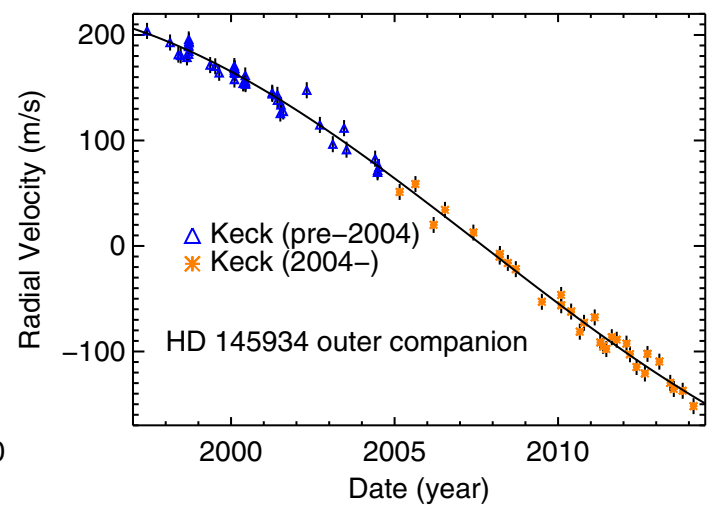

(d) HD 145934 outer companion

Figure 13. Radial velocity and Keplerian fit for HD 145934. Solid lines represent the best-fit Keplerian orbit. (a) Keck RVs overplotted by best-fit two-planet Keplerian model that includes a long-period companion to account for curvature. (b) Residuals of the RVs with the best-fit two-planet Keplerian model subtracted. (c) and (d): the RV curves for HD $145934 b$ and the outer long-period companion, respectively. 
These differences are all well within $1 \sigma$ of our presented set of parameters. We conclude that our modeling of the outer companion is sufficiently flexible to have no important effects on our estimates of the planet's orbital parameters.

Given that the rms of the residuals to the fit is $7.83 \mathrm{~m} \mathrm{~s}^{-1}$, we assume a stellar jitter of $7.5 \mathrm{~m} \mathrm{~s}^{-1}$ in our fit. Hekker et al. (2006) performed a survey of stable K giants with jitters lower than $20 \mathrm{~m} \mathrm{~s}^{-1}$. The most stable of that sample range between 6 and $15 \mathrm{~m} \mathrm{~s}^{-1}$, so our choice of jitter is reasonable and also consistent with the residuals. The residuals to the resulting bestfit Keplerian model have rms of $7.80 \mathrm{~m} \mathrm{~s}^{-1}$ and $\chi_{v}^{2}$ of 1.05 . We find that HD $145934 b$ has a period of $7.48 \pm 0.27 \mathrm{yr}$, an orbital eccentricity of $0.053_{-0.063}^{+0.053}$, and a semi-amplitude of $22.9 \pm 2.6 \mathrm{~m} \mathrm{~s}^{-1}$. The minimum mass of the planetary companion is $2.28 \pm 0.26 M_{\text {Jup }}$.

The presence of curvature in the binary companion's orbit implies that either it is highly eccentric and near periapse, or that we have observed a nonnegligible portion of its orbit. The latter is more likely, and implies that its orbital period is a several or dozens of decades, not millennia.

\section{DISCUSSION}

Our analysis of 13 exoplanets uses recent Keck-HIRES radial velocities and other published data. We see that there is need for follow-up work, as in the cases of GJ 849 and HD 145934 for better constraints and further analysis. In the instance of HD 66428, whose residuals show a previously unseen linear trend, we will monitor for the completion of orbits or to rule the companion out as a planet.

We have reduced the uncertainties in the parameters for many planets. The up-to-date HIRES data allowed us to place upper limits or constrain several orbits. From our sample, we identify two planets as Jupiter analogs around Sun-like due to similarities in semimajor axis (5.2 AU): HD $24040 b$ and HD $187123 c$, although both are much more massive than Jupiter, and the latter's orbit is somewhat eccentric. We have discovered a new planet, HD $145934 b$, and its host star's residuals show curvature whose velocity semiamplitude is indicative of a probable stellar or brown dwarf companion.

We confirm GJ $849 c$, and find that it is the planet with the longest known period around an M dwarf so far. GJ 849 is a rare system in that it is a multi-giant-planet system around an M-dwarf. In all of our multi-planet systems, the inner planet is less massive, though this fact is certainly influenced by the soft decrease in semiamplitude with orbital distance $\left(K \propto a^{-1 / 2}\right)$. HD 66428 may be a case where the planet's high eccentricity and the presence of a linear trend in the system are signs the outer companion has affected the inner planet's orbit, as Kane et al. (2014) found in the case of HD 4203.

All of these systems, but perhaps especially the "highly hierarchical" systems (Wright 2010) HD 187123 and HD 217107 , will be valuable for reconciling observations and the theory of planetary migration. These two systems are at present the only known examples of systems containing a hot Jupiter (gas giant with $P<10$ days and $M \sin i>0.1 M_{\text {Jup }}$ ) and a very-longperiod planet $(P>5 \mathrm{yr})$ with a well determined orbit. In both cases, the outer planet is $\sim 3$ times the mass of the inner planet, and there is no evidence of other planets in the system.

There are only two other systems with hot Jupiters and well-constrained long-period ( $P>1 \mathrm{yr}$ ) outer planets: HIP 14810 (Butler et al. 2006; Wright et al. 2009) and HAT-P-13 (Bakos et al. 2009; Winn et al. 2010). The former case remains anomalous in that the innermost planet is the most massive, with
$M \sin i=3.9 M_{\mathrm{Jup}}$ (the outermost planet has $M \sin i=0.6 M_{\mathrm{Jup}}$ and $P=2.6 \mathrm{yr}$; there is also a third, intermediate planet in the system). The latter case has an especially high mass ratio, having a highly eccentric $M \sin i>14 M_{\text {Jup }}$ outer planet and an inner, transiting planet with $M=0.86 M_{\text {Jup }}$. We know from both RV studies (Wright et al. 2009) and the Kepler results (Latham et al. 2011) that "hot Jupiters are lonely," at least when it comes to companions within $\sim 1$ AU. Continued long-term monitoring of other hot Jupiters will establish whether they have frequently have "cold friends" at larger orbital distances (e.g., Knutson et al. 2014).

We thank the many observers who contributed to the Lick and Keck-HIRES measurements reported here, especially John Johnson, Debra Fischer, Steven Vogt, and R. Paul Butler. We gratefully acknowledge the efforts and dedication of the Keck Observatory staff, especially Scott Dahm, Hien Tran, Grant Hill, and Gregg Doppmann for support of HIRES and Greg Wirth for support of remote observing.

We thank NASA, the University of California, and the University of Hawaii for their allocations of time on the Keck I telescope. Data presented herein were obtained at the W. M. Keck Observatory from telescope time allocated to the National Aeronautics and Space Administration through the agency's scientific partnership with the California Institute of Technology and the University of California. The Observatory was made possible by the generous financial support of the W. M. Keck Foundation. We wish to recognize and acknowledge the very significant cultural role and reverence that the summit of Mauna Kea has always had within the indigenous Hawaiian community. We are most fortunate to have the opportunity to conduct observations from this mountain.

We thank the many astronomers that contributed to the published CORALIE, ELODIE, SOPHIE, and HARPS RV measurements of these important long-period systems. In particular, we thank Xavier Bonfils for providing us with the HARPS radial velocity data of GJ 849 used in Bonfils et al. (2013).

The authors acknowledge the Pennsylvania State Research Computing and Cyberinfrastructure Group for providing computational resources and support that have contributed to the results reported within this paper.

This work was partially supported by: NASA Keck PI Data Awards, administered by the NASA Exoplanet Science Institute, including awards 2007B_N095Hr, 2010A_N147Hr, 2011A\&B_N141Hr, \& 2012A_N129Hr; NASA Origins of Solar Systems grant NNX09AB35G; NASA Astrobiology Institute grant NNA09DA76A; and the Center for Exoplanets and Habitable Worlds (which is supported by the Pennsylvania State University, the Eberly College of Science, and the Pennsylvania Space Grant Consortium). We acknowledge NSF grant AST-1211441.

This work has made use of data from the SIMBAD Astronomical Database (operated at CDS, Strasbourg, France); NASA's Astrophysics Data System Bibliographic Services; and of the Exoplanet Orbit Database and Exoplanet Data Explorer at exoplanets.org.

Facility: Keck:I

\section{REFERENCES}

Bakos, G. Á., Howard, A. W., Noyes, R. W., et al. 2009, ApJ, 707, 446 Baliunas, S. L., Donahue, R. A., Soon, W. H., et al. 1995, ApJ, 438, 269 Baluev, R. V. 2009, MNRAS, 393, 969

Baranne, A., Queloz, D., Mayor, M., et al. 1996, A\&AS, 119, 373 
Barnes, R., \& Raymond, S. N. 2004, ApJ, 617, 569

Bean, J. L., McArthur, B. E., Benedict, G. F., \& Armstrong, A. 2008, ApJ, 672,1202

Boisse, I., Pepe, F., Perrier, C., et al. 2012, A\&A, 545, A55

Bonfils, X., Delfosse, X., Udry, S., et al. 2013, A\&A, 549, A109

Bouchy, F. Sophie Team 2006, in Tenth Anniversary of 51 Peg-b: Status of and Prospects for Hot Jupiter Studies, ed. L. Arnold, F. Bouchy, \& C. Moutou (Paris: Fronties Group), 319

Burke, C. J., Bryson, S. T., Mullally, F., et al. 2014, ApJS, 210, 19

Butler, R. P., Marcy, G. W., Vogt, S. S., \& Apps, K. 1998, PASP, 110, 1389

Butler, R. P., Marcy, G. W., Williams, E., et al. 1996, PASP, 108, 500

Butler, R. P., Wright, J. T., Marcy, G. W., et al. 2006, ApJ, 646, 505

Chambers, J. E. 1999, MNRAS, 304, 793

Cochran, W. D., \& Hatzes, A. P. 1994, Ap\&SS, 212, 28

Crepp, J. R., Johnson, J. A., Howard, A. W., et al. 2012, ApJ, 761, 39

Crepp, J. R., Johnson, J. A., Howard, A. W., et al. 2013a, ApJ, 771, 46

Crepp, J. R., Johnson, J. A., Howard, A. W., et al. 2013b, ApJ, 774, 1

Crepp, J. R., Johnson, J. A., Howard, A. W., et al. 2014, ApJ, 781, 29

Deming, D., Espenak, F., Jennings, D. E., Brault, J. W., \& Wagner, J. 1987, ApJ, 316,771

Dravins, D. 1985, in Stellar Radial Velocities, ed. A. G. D. Philip \& D. W. Latham (Schenectady, NY: L. Davis Press), 311

Dumusque, X., Lovis, C., Ségransan, D., et al. 2011, A\&A, 535, A55

Endl, M., Robertson, P., Cochran, W. D., et al. 2012, ApJ, 759, 19

Fischer, D. A., Marcy, G. W., Butler, R. P., Vogt, S. S., \& Apps, K. 1999, PASP, 111,50

Fischer, D. A., Marcy, G. W., Butler, R. P., et al. 2001, ApJ, 551, 1107

Fischer, D. A., Marcy, G. W., \& Spronck, J. F. P. 2014, ApJS, 210, 5

Ford, E. B. 2006, ApJ, 642, 505

Gomes da Silva, J., Santos, N. C., Bonfils, X., et al. 2012, A\&A, 541, A9

Han, E., Wang, S. X., Wright, J. T., et al. 2014, PASP, 126, 827

Hekker, S., Reffert, S., Quirrenbach, A., et al. 2006, A\&A, 454, 943

Horne, J. H., \& Baliunas, S. L. 1986, ApJ, 302, 757

Howard, A. W., Johnson, J. A., Marcy, G. W., et al. 2009, ApJ, 696, 75

Howard, A. W., Johnson, J. A., Marcy, G. W., et al. 2010, ApJ, 721, 1467

Howard, A. W., Johnson, J. A., Marcy, G. W., et al. 2011a, ApJ, 726, 73

Howard, A. W., Johnson, J. A., Marcy, G. W., et al. 2011b, ApJ, 730, 10

Howard, A. W., Marcy, G. W., Johnson, J. A., et al. 2010, Sci, 330, 653

Isaacson, H., \& Fischer, D. 2010, ApJ, 725, 875

Johnson, J. A., Howard, A. W., Marcy, G. W., et al. 2010, PASP, 122, 149

Kane, S. R., Howell, S. B., Horch, E. P., et al. 2014, ApJ, 785, 93

Knutson, H. A., Fulton, B. J., Montet, B. T., et al. 2014, ApJ, 785, 126

Latham, D. W., Rowe, J. F., Quinn, S. N., et al. 2011, ApJL, 732, L24

Lovis, C., Dumusque, X., Santos, N. C., et al. 2011, arXiv:1107.5325

Macintosh, B., Graham, J. R., Ingraham, P., et al. 2014, PNAS, 111,12661

Marcy, G. W., Butler, R. P., Fischer, D. A., et al. 2002, ApJ, 581, 1375

Marcy, G. W., Butler, R. P., Vogt, S. S., et al. 2005, ApJ, 619, 570

Markwardt, C. B. 2009, in ASP Conf. Ser. 411, Astronomical Data Analysis Software and Systems XVIII, ed. D. A. Bohlender, D. Durand, \& P. Dowler (San Francisco, CA: ASP), 251

Marmier, M., Ségransan, D., Udry, S., et al. 2013, A\&A, 551, A90

Mayor, M., Pepe, F., Queloz, D., et al. 2003, Msngr, 114, 20

Mayor, M., \& Queloz, D. 1995, Natur, 378, 355
Meschiari, S., Laughlin, G., Vogt, S. S., et al. 2011, ApJ, 727, 117

Montet, B. T., Crepp, J. R., Johnson, J. A., Howard, A. W., \& Marcy, G. W. 2014, ApJ, 781, 28

Naef, D., Mayor, M., Beuzit, J. L., et al. 2004, A\&A, 414, 351

Naef, D., Mayor, M., Pepe, F., et al. 2001, A\&A, 375, 205

Nelson, B., Ford, E. B., \& Payne, M. J. 2014, ApJS, 210, 11

Önehag, A., Heiter, U., Gustafsson, B., et al. 2012, A\&A, 542, A33

Patel, S. G., Vogt, S. S., Marcy, G. W., et al. 2007, ApJ, 665, 744

Press, W. H., Teukolsky, S. A., Vetterling, W. T., \& Flannery, B. P. 2002, Numerical Recipes in C: The Art of Scientific Computing (2nd ed.; New York: Cambridge Univ. Press)

Queloz, D., Mayor, M., Weber, L., et al. 2000, A\&A, 354, 99

Rojas-Ayala, B., Covey, K. R., Muirhead, P. S., \& Lloyd, J. P. 2012, ApJ, 748, 93

Saar, S. H., Butler, R. P., \& Marcy, G. W. 1998, in ASP Conf. Ser. 154, Cool Stars, Stellar Systems, and the Sun, ed. R. A. Donahue \& J. A. Bookbinder (San Francisco, CA: ASP), 1895

Santos, N. C., Gomes da Silva, J., Lovis, C., \& Melo, C. 2010, A\&A, 511, AA54

Scargle, J. D. 1982, ApJ, 263, 835

Takeda, G., Ford, E. B., Sills, A., et al. 2007, ApJS, 168, 297

Terrien, R. C., Mahadevan, S., Bender, C. F., et al. 2012, ApJL, 747, L38

Torres, G., Andersen, J., \& Giménez, A. 2010, A\&ARv, 18, 67

Tull, R. G. 1998, Proc. SPIE, 3355, 387

Tull, R. G., MacQueen, P. J., Sneden, C., \& Lambert, D. L. 1995, PASP, 107,251

Valenti, J. A., \& Fischer, D. A. 2005, ApJS, 159, 141

van Leeuwen, F. 2007, in Astrophysics and Space Science Library (Vol. 350; Berlin: Springer)

Vogt, S. S. 1987, PASP, 99, 1214

Vogt, S. S., Allen, S. L., Bigelow, B. C., et al. 1994, Proc. SPIE, 2198, 362

Vogt, S. S., Butler, R. P., Marcy, G. W., et al. 2005, ApJ, 632, 638

Walker, G. A. H., Walker, A. R., Irwin, A. W., et al. 1995, Icar, 116, 359

Wang, X., Sharon, Wright, J. T., Cochran, W., et al. 2012, ApJ, 761, 46

Wetherill, G. W. 1994, Ap\&SS, 212, 23

Winn, J. N., Johnson, J. A., Howard, A. W., et al. 2010, ApJ, 718, 575

Wittenmyer, R. A., Endl, M., Cochran, W. D., Levison, H. F., \& Henry, G. W. 2009, ApJS, 182, 97

Wright, J. T. 2005, PASP, 117, 657

Wright, J. T. 2006, PhD thesis, Univ. California, Berkeley

Wright, J. T. 2010, in Extrasolar Planets in Multi-Body Systems: Theory and Observations, ed. K. Goździewski, A. Niedzielski, \& J. Schneider (EAS Publication Series, Vol. 42; Cambridge: Cambridge Univ. Press), 3

Wright, J. T., Fischer, D. A., Ford, E. B., et al. 2009, ApJL, 699, L97

Wright, J. T., \& Gaudi, B. S. 2013, Planets, Stars and Stellar Systems, Volume 3: Solar and Stellar Planetary Systems, ed. T. D. Oswalt, L. M. French, M. Linda, \& P. kalas (Dordrecht: Springer), 489

Wright, J. T., \& Howard, A. W. 2009, ApJS, 182, 205

Wright, J. T., Marcy, G. W., Butler, R. P., \& Vogt, S. S. 2004, ApJS, 152,261

Wright, J. T., Marcy, G. W., Butler, R. P., et al. 2008, ApJL, 683, L63

Wright, J. T., Marcy, G. W., Fischer, D. A., et al. 2007, ApJ, 657, 533

Wright, J. T., Upadhyay, S., Marcy, G. W., et al. 2009, ApJ, 693, 1084

Wright, J. T., Veras, D., Ford, E. B., et al. 2011, ApJ, 730, 93 